
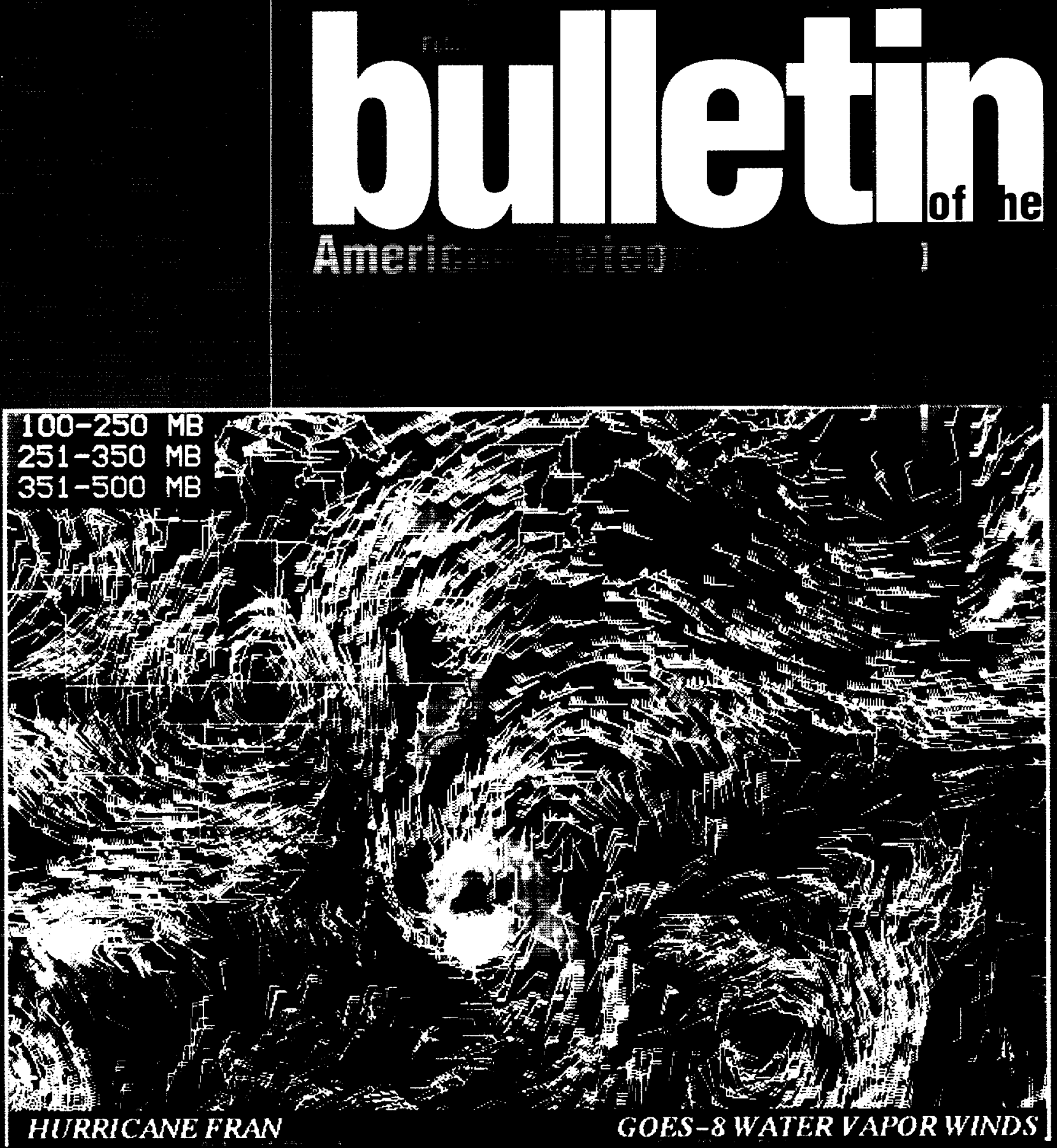



\title{
Upper-Tropospheric Winds Derived from Geostationary Satellite Water Vapor Observations
}

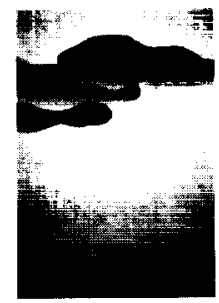

\author{
Christopher S. Velden, ${ }^{*}$ Christopher M. Hayden, ${ }^{+}$Steven J. Nieman, ${ }^{*}$ \\ W. Paul Menzel," Steven Wanzong, ${ }^{*}$ and James S. Goerss ${ }^{@}$
}

\begin{abstract}
The coverage and quality of remotely sensed upper-tropospheric moisture parameters have improved considerably with the deployment of a new generation of operational geostationary meteorological satellites: $G O E S-8 / 9$ and $G M S-5$. The GOES $-8 / 9$ water vapor imaging capabilities have increased as a result of improved radiometric sensitivity and higher spatial resolution. The addition of a water vapor sensing channel on the latest GMS permits nearly global viewing of upper-tropospheric water vapor (when joined with GOES and Meteosat) and enhances the commonality of geostationary meteorological satellite observing capabilities. Upper-tropospheric motions derived from sequential water vapor imagery provided by these satellites can be objectively extracted by automated techniques. Wind fields can be deduced in both cloudy and cloud-free environments. In addition to the spatially coherent nature of these vector fields, the GOES-8/9 multispectral water vapor sensing capabilities allow for determination of wind fields over multiple tropospheric layers in cloud-free environments. This article provides an update on the latest efforts to extract water vapor motion displacements over meteorological scales ranging from subsynoptic to global. The potential applications of these data to impact operations, numerical assimilation and prediction, and research studies are discussed.
\end{abstract}

\section{Introduction}

Two of the most important challenges facing the atmospheric remote sensing community today are 1) transforming information-wealthy imagery into quantitative parameters and 2) ensuring these parameters advance our understanding and prediction of meteorological phenomena beyond existing methods of observing the atmosphere. The latter point is of particular significance given the advances in numerical weather prediction (NWP) over the past decade. Further improvements in prediction will partially rely on superior measure-

*Cooperative Institute for Meteorological Satellite Studies, Madison, Wisconsin.

+National Environmental Satellite, Data and Information Service, NOAA, Madison, Wisconsin.

"Advanced Satellite Products Project, NOAA NESDIS, Madison, Wisconsin.

${ }^{(a)}$ Naval Research Laboratory, Monterey, California. Corresponding author address: Christopher Velden, UWCIMSS, 1225 West Dayton St., Madison, WI 53706.

E-mail: chrisv@ssec.wisc.edu

In final form 3 September 1996.

(1)1997 American Meteorological Society ments of the initial atmospheric state and optimized assimilation of these measurements. This is of particular relevance to remotely sensed observations when considering regions of the globe that are traditionally void of conventional meteorological data.

Water vapor radiance measurements from geostationary satellites have been used extensively in a qualitative mode by analysts and forecasters (Weldon and Holmes 1991). Early attempts to quantify the data by employing manual methods to track moisture features in animated image sequences met with modest success (Hayden and Stewart 1987). More recent studies have demonstrated the ability of automated extraction techniques to produce spatially coherent water vapor wind vector (WVWV) fields comparable in quality to operational cloud-tracked winds (Laurent 1993; Velden 1993; Holmlund 1993).

Development of automated wind-extraction algorithms at the University of Wisconsin-Madison Cooperative Institute for Meteorological Satellite Studies (CIMSS) has progressed to the stage of operational implementation (Nieman et al. 1997). In collaboration with the National Oceanic and Atmospheric Administration (NOAA) National Environmental Satellite 
Data and Information Service (NESDIS), efforts at CIMSS have focused on the research, development, and demonstration of these algorithms toward the goal of routine inclusion of the WVWV measurements into the operational data stream and numerical weather prediction. Attributable to the advances in computing power, algorithm automation, and communications, high-density WVWV fields from multiple geostationary satellites each covering near full-disk viewing are achievable in a time frame and frequency commensurate with operational requirements.

This article describes the general characteristics of satellite-derived WVWV, the methodology employed at CIMSS to derive the vector fields, and the currently attainable accuracies. Several examples of applications will be presented, as well as initial numerical model assimilation impact results. This is followed by a brief discussion of the potential benefits to the observing system, the limitations and challenges in effective use of the data, and future prospects.

\section{Background}

The quest for quantitative wind information over oceanic regions lacking in conventional observations was the prime motivation for the development of methods to track clouds in sequential satellite imagery. These "cloud-drift winds" became an important component of the global observing system during the early 1980s. However, an obvious limitation of this product resulted from the fact that vast cloud-free regions were left unsampled. About this time, $6-7-\mu \mathrm{m}$ water vapor absorption band channels became available on geostationary satellites (Meteosat and GOES). Imagery from these observations revealed atmospheric motion even in cloud-free areas, and research with these data led Eigenwillig and Fischer (1982) to conclude that feature tracking was possible within a reasonable accuracy. This was supported in a study by Stewart et al. (1985) using GOES imagery and a state-of-the-art tracking system (McIDAS, Mancomputer Interactive Data Access System).

These early attempts at WVWV employed basic techniques similar to cloud tracking, in which a human operator selected a target feature in an image and subjectively followed it through subsequent imagery to determine a displacement. The heights were crudely assigned utilizing basic radiative transfer formulae to transform water vapor (WV) pixel radiance to a brightness temperature, which was then matched to a collocated temperature profile (model guess or climatology). While WVWV fields were successfully demonstrated (LeMarshall et al. 1985; Hayden and Stewart 1987), the subjectivity and labor-intensive nature of the technique prevented the product from becoming fully operational.

In the late 1980s and early 1990s, advancement in the automation of satellite feature tracking techniques (Merrill et al. 1991) was partially successful toward easing the production constraints on WVWV fields. However, the quality control (QC) of the WVWV fields still demanded intensive manual intervention, which restricted operational utility. In addition, numerical model forecast accuracies were increasing rapidly, and the quality of the GOES-7 WVWV observations was insufficient to make significant impacts (Velden 1990). By contrast, the European community (European Space Operations Center) developed an automated WVWV extraction algorithm using higher quality Meteosat observations. This product became operational in 1993, although the QC is very strict (Laurent 1993; Holmlund 1995).

Recognizing the need for improved imaging and sounding capabilities from geostationary orbit, NOAA began development of its next generation of satellites, GOES I-M, in 1985 (Menzel and Purdom 1994). The first two of these GOES were successfully deployed in 1994 and 1995. This new generation features advanced sensors enabling improved spatial resolution and signal-to-noise ratios. Specifically, the water vapor channels (imager and sounder) are a significant upgrade from the previous GOES (Table 1). A qualitative comparison of GOES-7 and GOES-8 water vapor imagery is shown in Fig. 1 and illustrates the signal-to-noise improvements associated with the new generation.

The remainder of this article will focus on the current ability to extract multispectral (imager and sounder) WVWV information from geostationary satellites. It will be shown that the combination of improved radiometric observations, advanced and automated wind extraction algorithms, and an optimized processing strategy can lead to a product that has the potential to make an important contribution to the upper-tropospheric observing system. The WVWV applications cover meteorological scales ranging from meso- to climate and can benefit both research and operations.

\section{Methodology}

The procedure for deriving WVWV at CIMSS parallels the basic technique employed for cloud track- 
ing. The fully automated algorithm is housed within McIDAS-X (UNIX) architecture. A description of the system can be found in Merrill (1989) and Merrill et al. (1991), with the latest upgrades (including specialized image registration) in Nieman et al. (1997). Only a brief account will be given here, with attention given to features unique to the derivation of WVWV.

\section{a. Tracer selection and tracking metric}

The traditional selection process for targeting features in the case of

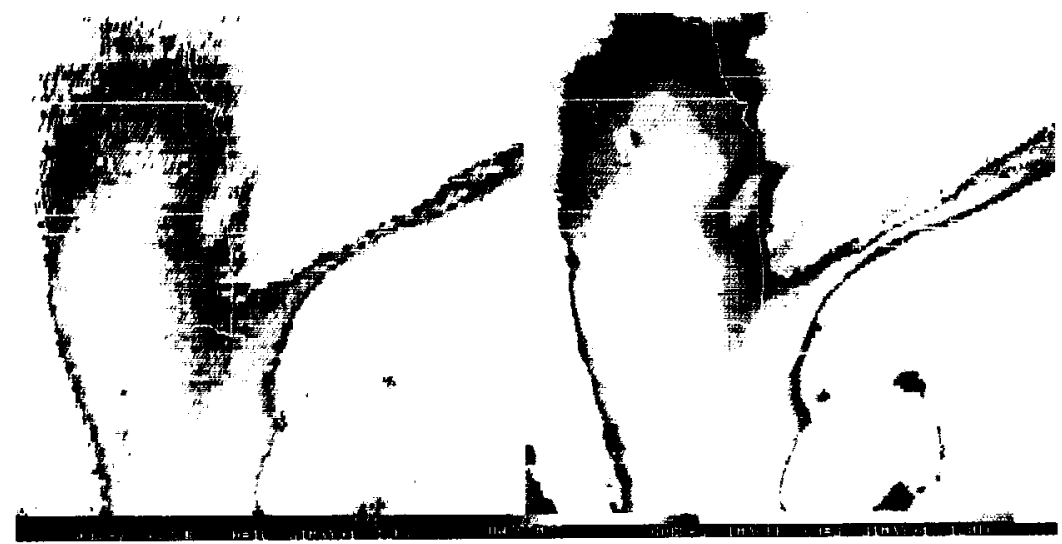

FIG. 1. 6.7- $\mu \mathrm{m}$ water vapor imagery over the south-central United States from GOES-7 (left) and GOES-8 (right) at 1300 UTC 31 May 1994. The color enhancement reflects the brightness temperature range with stronger yellows indicating drier air. tracking clouds is based on the isola-

tion of the lowest (cloud) brightness temperatures (BT) within a pixel array (target box) of a size selected by the operator. Local bidirectional gradients are computed and compared with empirically determined thresholds to identify those features exhibiting sufficient variability to be classified as targets (Merrill 1989).

Water vapor structure in cloud-free environments demands a different strategy. Features exhibiting the strongest gradient profiles may not be confined to the coldest BT, as in clouds. In the CIMSS algorithm, targets are identified by evaluating the bidirectional gradients surrounding each pixel in the target array and selecting the maximum value (exceeding thresholds). The operator can control the density of targets by selecting the size of the target box and setting the gradient thresholds. This flexibility is important when considering different meteorological scales to be measured and for computational efficiency.

In practice, WVWV targets are selected in both cloudy and cloud-free regions. Experience has shown that the density of higher-quality targets is greater in cloudy and/or very moist regimes exhibiting high contrast. This is reflected in the resulting WVWV wind field characteristics, which are discussed in the next section.

The tracking metric currently employs a simple search for the minimum in the sum of squares of the radiance differences between the target and search arrays in subsequent images (usually two subsequent images at 30-min intervals). Fully cross-correlational algorithms are more computationally demanding but are being investigated. A model guess forecast of the upper-level wind is employed to economize on the search areas. In practice, two subsequent images are utilized with the target image to derive two displacement vectors. If the vectors survive consistency checks, an average is calculated and becomes the rep-

TABLE 1. Performance specifications and spatial resolution of current geostationary satellite water vapor channels. NEDT is the noise equivalent temperature difference $(\mathrm{K})$ evaluated at $230 \mathrm{~K}$. Numbers in parentheses indicate central wavelengths.

\begin{tabular}{|c|c|c|}
\hline & NEDT & Resolution (km) \\
\hline \multicolumn{3}{|l|}{ Imagers } \\
\hline GOES-9 (6.7) & 0.11 & 8 \\
\hline GOES-8 (6.7) & 0.22 & 8 \\
\hline GOES-7 (6.7) & 1.00 & 14 \\
\hline Meteosat-5 (6.5) & 0.40 & 5 \\
\hline GMS-5 (6.9) & 0.40 & 5 \\
\hline \multicolumn{3}{|l|}{ Sounders } \\
\hline GOES-9 (7.3) & 0.33 & 8 \\
\hline GOES-9 (7.0) & 0.27 & 8 \\
\hline GOES-8 (7.3) & 0.51 & 8 \\
\hline GOES-8 $(7.0)$ & 0.40 & 8 \\
\hline GOES-7 (7.0) & 3.70 & 14 \\
\hline
\end{tabular}


resentative WVWV. Experimentation has shown that 30-min interval imagery is slightly superior to hourly intervals for optimal WVWV tracking.

\section{b. Height assignment and quality control}

A long-standing concern regarding WVWV is the height assignment. Particularly in cloud-free regions, the radiometric signal from pure water vapor structures is a result of emittance over a finite layer. This can be complicated further by radiance contributions from multiple moist layers (Weldon and Holmes 1991). The challenge is to assign a height level that best represents the motion of the feature being tracked.

The strategy employed in the CIMSS algorithm for height assignment is a two-step approach. The first is the conversion of instrument measured radiances into BT. This requires an accurate definition of the spectral response of the instrument. Figure 2 shows the spectral response functions for GOES-8, Meteosat-5, and $G M S-5$ water vapor channels. It is important to note that the different instruments are sensing radiation from slightly different parts of the $\mathrm{H}_{2} \mathrm{O}$ absorption band. Sampling the center of the absorption band (around $6.3 \mu \mathrm{m}$ ) yields radiation from the upper levels of the troposphere (i.e., radiation from below has already been absorbed by the water vapor). Sampling away from the center of the absorption band yields radiation from lower levels of the troposphere. Figure 3 shows the weighting functions (which denote energy contribution profiles) for the GOES- 8 imager and sounder $\mathrm{H}_{2} \mathrm{O}$ sensitive bands, as well as the Meteosat-5 and GMS-5 water vapor bands. The

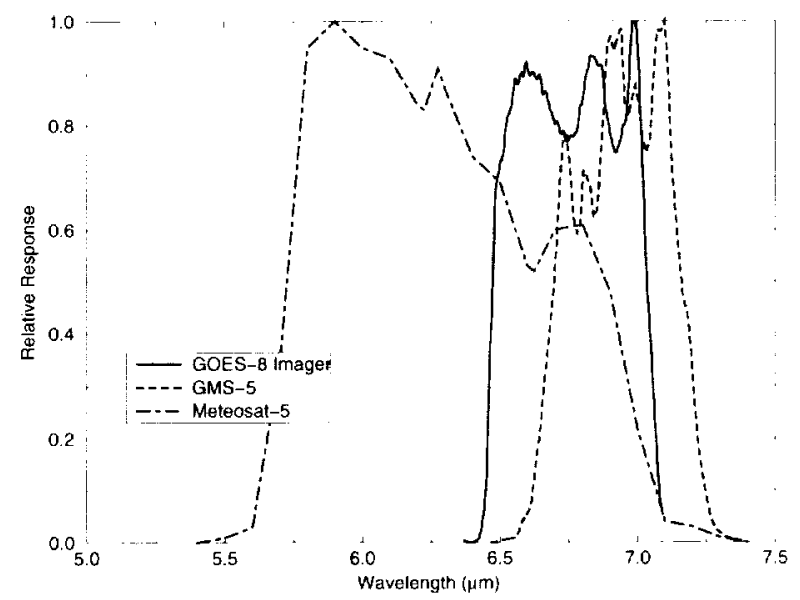

FIG. 2. Water vapor channel spectral response functions for GOES-8, Meteosat-5, and GMS-5. The scale at the left indicates a normalized measure of the relative energy contribution per spectral region.
GOES- 8 sounder $7.3-\mu \mathrm{m}$ band senses deeper into the atmosphere than the other bands, as it is farther away from the $\mathrm{H}_{2} \mathrm{O}$ absorption band center. The GMS-5 $6.9-\mu \mathrm{m}$ band senses a similar layer of the atmosphere as the GOES-8 sounder $7.0-\mu \mathrm{m}$ band.

Once a target has been selected for tracking, the BT in the target area are averaged and this value is matched with a collocated model guess temperature profile. The level of optimum fit is used to assign an initial pressure height. As indicated in Fig. 3, these heights are typically within the range of $250-600 \mathrm{mb}$.

The second step of the height assignment procedure involves an important and unique component of the CIMSS winds processing system and is automatically
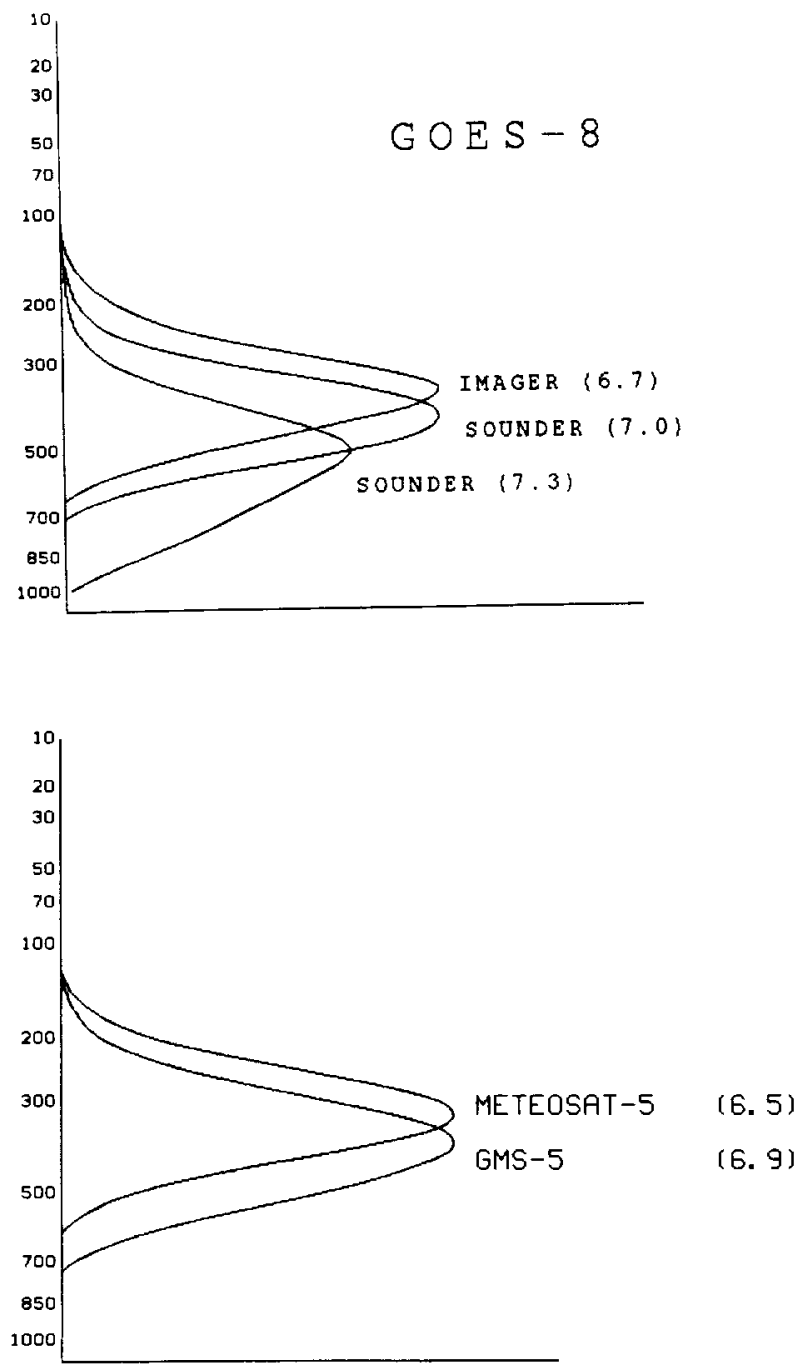

FIG. 3. Weighting functions associated with geostationary satellite water vapor channels in a typical subtropical atmosphere: GOES-8 (top), Meteosat-5 and GMS-5 (bottom). Numbers in parentheses refer to central wavelengths. Peaks in the functions correspond to tropospheric layers of greatest energy contribution. 
invoked following the completed computation of the WVWV field. The initial WVWV heights are reevaluated as part of the postprocessing algorithm (objective QC procedure outlined below). The basic rationale for this height reassessment is to seek the assignment of a level that best fits other multivariate information from conventional data, neighboring WVWV and cloud-tracked winds, numerical model output, etc. In this sense, the procedure has an element of data assimilation (Hayden and Velden 1991).

The postprocessing algorithm involves a two-stage, three-dimensional objective analysis (Hayden and Purser 1995) of the wind field using background information from a numerical model forecast. The procedure is schematically shown in Fig. 4 . The objective analysis employed is an adaptation of the recursive filter (RF) analysis described by Hayden and Purser (1988). It is a basic successive approximation method but contains a unique feature of locally varying scaling, which gives it greater flexibility over nonhomogeneous data. The general properties of the $\mathrm{RF}$, and its relationship to a characteristic scale, are given in Hayden and Purser (1995).

The first stage of the postprocessing algorithm provides a preliminary wind analysis using the WVWV at their initially assigned pressures, any ancillary reports (i.e., cloud-drift winds), and pseudodata from a numerical model 6- or 12-h forecast. At this point, an adjustment to the WVWV speed is applied based on the well-known slow bias in upper-tropospheric cloud-drift winds. It is suspected that this bias is a result of clouds and water vapor motions not being a perfect indicator of the instantaneous wind, especially in higher wind regimes. This slow bias is mitigated by an empirically determined correction term (partly based on statistics from the European Centre for Medium-Range Weather Forecasts), which is applied to WVWV with speeds greater than $10 \mathrm{~m} \mathrm{~s}^{-1}$. The adjustment increments each vector by $8 \%$ of vector speed.

The WVWV heights are then adjusted by conducting a vertical search aimed at minimizing a simple variational penalty function evaluated using the initial wind analyses and the model forecast:

$$
\begin{gathered}
B_{m, k}=\left(\frac{V_{m}-V_{i, j, k}}{F_{v}}\right)^{2}+\left(\frac{T_{m}-T_{i, j, k}}{F_{t}}\right)^{2}+\left(\frac{P_{m}-P_{i, j, k}}{F_{p}}\right)^{2} \\
+\left(\frac{d d_{m}-d d_{i, j, k}}{F_{d d}}\right)^{2}+\left(\frac{s_{m}-s_{i, j, k}}{F_{s}}\right)^{2},
\end{gathered}
$$

where $V=$ velocity, $T=$ temperature, $P=$ pressure, $d d=$ direction, and $s=$ speed. Here, subscript $m$ refers to a WVWV measurement, $i$ and $j$ are horizontal dimensions in the analysis, and $k$ is the vertical level. The denominators $F_{x}$ are weights defined by the operator that may be varied to emphasize the vector velocity, temperature (target BT), pressure, direction, and speed terms in the penalty function. The vertical search for a best fit (and hence, the maximum allowable reassignment) is limited to $\pm 150 \mathrm{mb}$ from the initial assignment. The height reassignment may fail if no minimum is found or if the minimum exceeds empirically determined thresholds. In these instances the vector is rejected.

The full RF analysis is repeated in stage two of the postprocessing algorithm using the reassigned and accelerated WVWV and output of the first analysis as a background field. The output of this analysis provides quality estimates (flags) for each vector based on the local quality of the analysis and the fit of the observation to that analysis. Vectors that do not attain an empirically determined threshold quality are rejected.

In regard to the threshold, a trade-off exists in the choice of the maximum allowable difference between the background field (which may be highly influenced by the model guess winds) and the vectors: if only a small difference is allowed, potential vector information is reduced; if a large difference is allowed, potential information content is increased but so are potential errors. The threshold currently employed in the operational NESDIS algorithm (a version of the CIMSS algorithm) is rather conservative and is based on an error analysis against collocated rawinsonde information. However, this threshold is not optimal in meteorological situations subject to difficult model

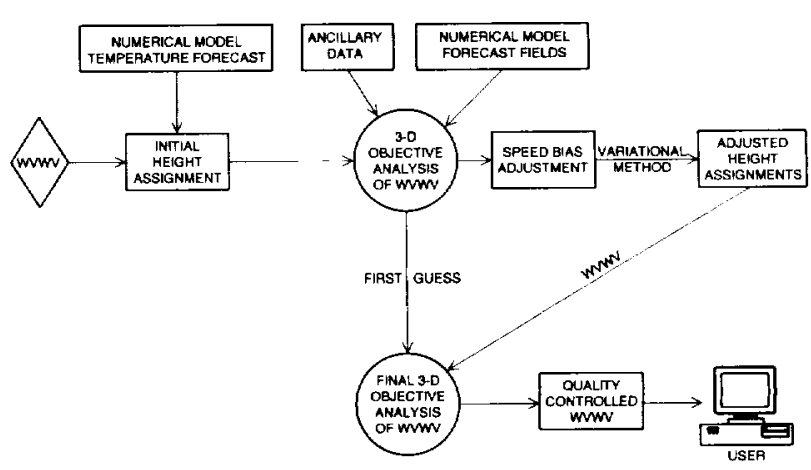

Fig. 4. Schematic of the objective quality control procedure employed in the CIMSS automated water vapor wind vector (WVWV) processing algorithm. 
forecasts, such as hurricanes or strong extratropical events. In this regard, a wide variety of options are available for regulating the analysis (such as the background field weights), the penalty function, and the final quality estimates. For WVWV, these options have been empirically tuned over several years of experimenting and application.

The precision attainable from current geostationary satellite water vapor channels allows WVWV to be processed at high density, providing a uniform, spatially coherent field of coverage. With GOES, WVWV from two sounder channels can be added to the analysis, resulting in augmentation of threedimensional coverage (see next section). To take full advantage of this coverage, the postprocessing algorithm is tunable in the following ways: 1) the objective analysis horizontal resolution (increment) can be increased to capture finer-scale features, 2) the model background field can be downweighted in the analysis, 3) a tighter fit of each WVWV to the analysis can be demanded due to the abundance of "neighbor checking," 4) added weight can be given to the velocity term in the penalty function, 5) vertical coupling requirements in the three-dimensional analysis can be made less strict, and 6) the rejection criteria based on the final quality flags can be relaxed. The final point indicates confidence in the ability of the WVWV fields to capture and retain details not present in the model forecast background field.

The result of this postprocessing strategy is an objectively based, quality-controlled WVWV field with vector heights assigned to pressure levels based on both radiometric properties and optimum fit with ancillary information. While in nature it is rare that a particular single level will perfectly represent a layer, our analysis of WVWV height assignment quality compared with collocated rawinsondes generally indicates good agreement and placement confidence within $50 \mathrm{mb}$ in most cases. WVWV accuracies and verification statistics are presented in section 5 .

\section{WVWV characteristics}

An example of the attainable coverage from GOES-86.7- $\mu \mathrm{m}$ WVWV is shown in Fig. 5. This field was produced using the CIMSS algorithm on a desktop workstation in about $10 \mathrm{~min}$. The coverage domain in this example represents a small portion of what is possible from geostationary viewing. Featured is Tropical Storm Pablo and its environment. The
WVWV have been processed at high density, using the tuned QC strategy described above. Several properties of the WVWV product are apparent from this example: 1) the coverage is uniform and spatially coherent, 2) the vertical distribution of information is spread over the $150-500-m b$ portion of the troposphere, 3) large-scale and synoptic features are well represented, and 4) smaller-scale features such as the storm outflow and adjacent environmental features are captured.

The last point is of particular importance, as this scale is often undersampled by the existing observational network and as a result is frequently not well represented in global objective analyses. For example, the narrow band of high-level 50-60-kt westerly winds just upstream (west) of Pablo was not captured by any operationally available data and not analyzed by the National Centers for Environmental Prediction (NCEP) operational global model. Pablo was moving steadily to the west and forecasted to slowly intensify. However, as Pablo encountered these winds over the next $24 \mathrm{~h}$, the strong shear disrupted the storm circulation and Pablo was subsequently downgraded to a depression. This illustrates how the WVWV can be utilized in a qualitative mode, since knowledge of the impending hostile upper-level environment could have played a role in the intensity forecast.

The vertical distribution of WVWV is dependent on several key factors: 1) characteristics of the spectral channel, such as central wavelength and band width; 2) airmass characteristics (including the amount of cloudiness and vertical profile of moisture); 3) season (less moisture in the cool season leads to lower mean heights); and 4) latitude (higher moisture content and tropopause in the Tropics leads to higher mean heights). In regard to the first point, the current operational geostationary weather satellites with water vapor channels (GOES, Meteosat, and GMS) have similar traits (Fig. 3 and Table 1), which is advantageous for global data assimilation and future climate research studies.

In addition to the $6.7-\mu \mathrm{m}$ channel on the imager, GOES-8/9 house an atmospheric sounder that includes two water vapor absorption channels centered at 7.0 and $7.3 \mu \mathrm{m}$ (Menzel and Purdom 1994). These sounder channels can be employed as surrogate imagers to track water vapor features radiating from lower layers of the troposphere (Fig. 3). An example of the WVWV coverage attainable from these two channels (GOES-8) is illustrated in Fig. 6. Of note is 
the spatial coherency in the WVWV fields derived from each channel. A histogram analysis of WVWV heights for this case is shown in Fig. 7. The heights are partitioned into 12.5 -mb bins and graphed to show the vertical distribution of WVWV for the 6.7- (imager channel 3), 7.0- (sounder channel 11), and 7.3(sounder channel 10) $\mu \mathrm{m}$ channels. Quasi-independent peaks in information are found in discrete layers; near $250 \mathrm{mb}$ (cloud), 300-350 mb (channel 3), 350$450 \mathrm{mb}$ (channel 11 ), and 450-550 mb (channel 10). While there is some redundancy in terms of coverage in the vertical, additional information is gained from the sounder channels, especially in the midtropospheric cloud-free regions. Integration of the sounder WVWV is important to the objective, three-dimensional analysis, which is part of the internal quality control discussed in the previous section. The sounder coverage domain is restricted due to observing constraints (Menzel and Purdom 1994); however, a programmable scanning strategy allows the sounder to view targeted areas of meteorological interest.

The coverage attainable from WVWV is quite uniform in most cases, since the observed displacement of a large portion of the targeted features is a result of pure moisture advection and adequately represents the instantaneous wind (after the bias correction). The coverage is especially complete in the summertime Tropics due to the presence of abundant moisture and other characteristics mentioned previously. There are, however, situations when extraction of useful WVWV information is difficult, if not impossible. These situations are summarized as follows: 1) very dry air masses lacking in moisture features, which prohibits good target selection; 2) regions of strong vertical motion, in which a coherent radiance signal can be significantly attenuated over the tracking interval; 3) uniform moisture fields lacking in contrast (flat gra-

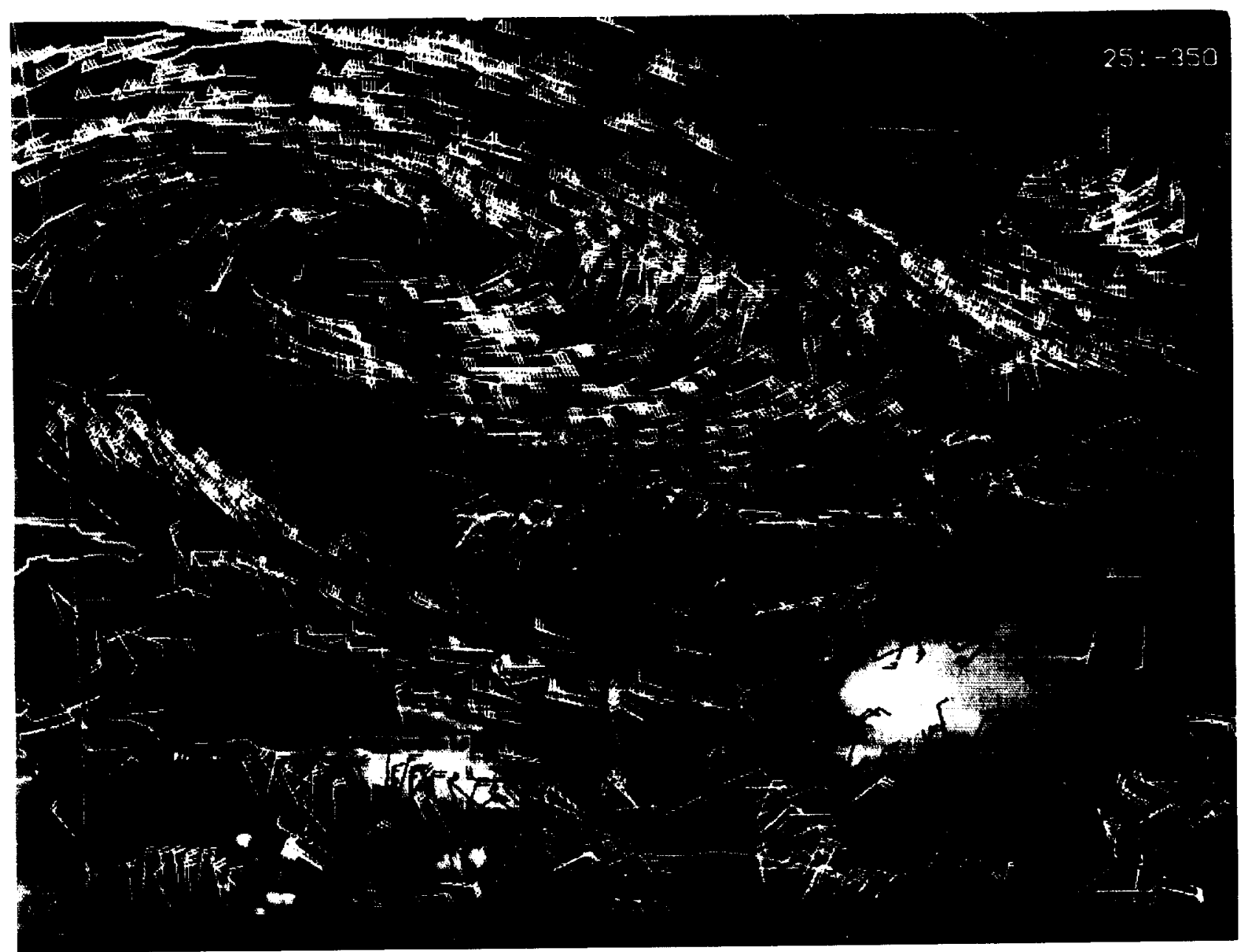

FIG. 5. High-density GOES-8 water vapor winds (6.7- $\mu \mathrm{m}$ channel) during Tropical Storm Pablo (lower right) on 7 October 1995. Vectors are in $\mathrm{kt}$ and colors refer to assigned heights $(\mathrm{mb})$ with the legend in the upper right-hand corner. 


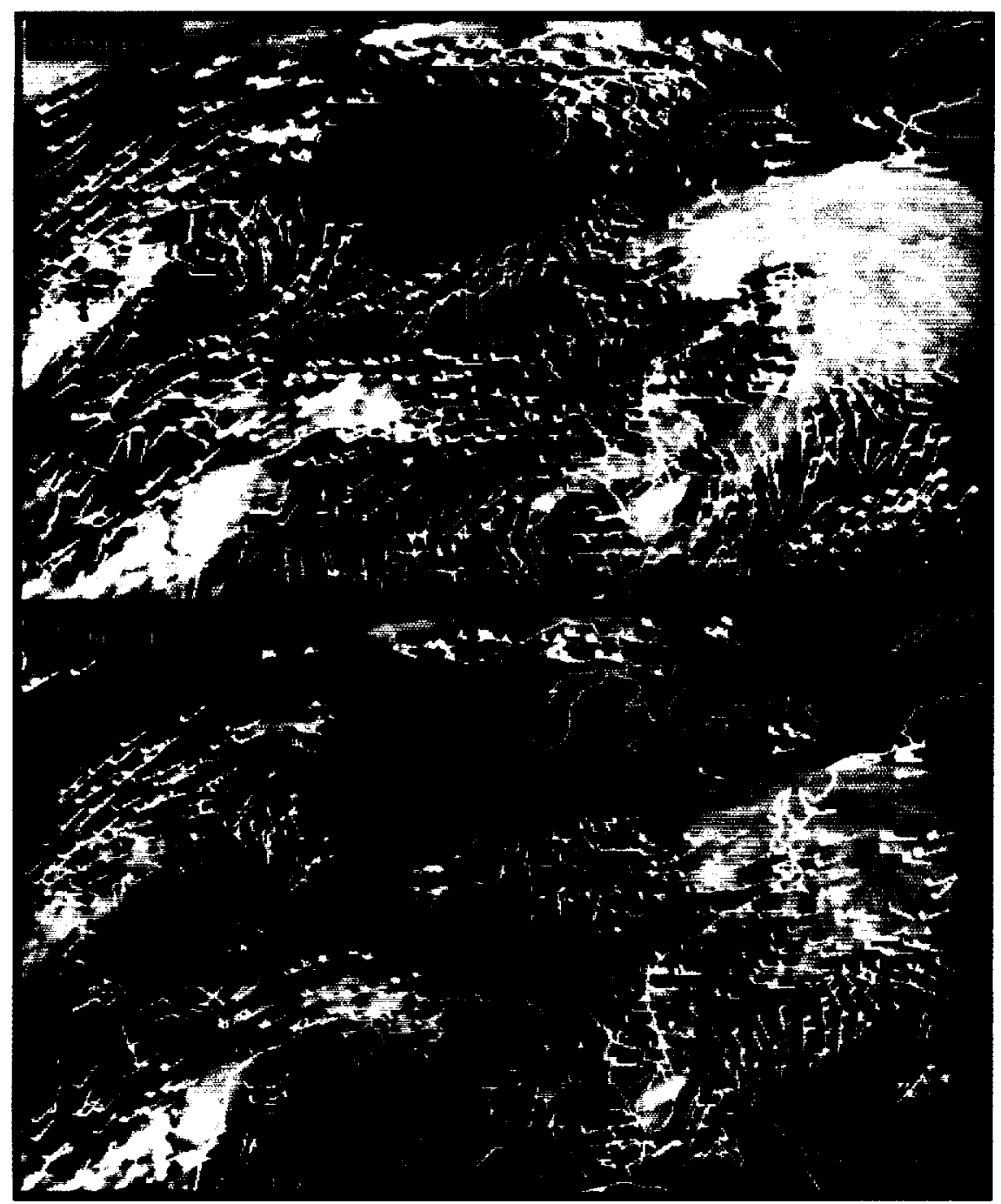

Fig. 6. Example of wind coverage that can be achieved from tracking features in the 7.0- and 7.3- $\mu \mathrm{m}$ channels from the GOES-8 sounder. Yellow (beige) vectors (kt) indicate winds with assigned heights above (below) $400 \mathrm{mb}$. The winds are plotted over their respective channels.

dients); 4) strong vertical shear zones, which can deform targets over the tracking interval; and 5) rapidly amplifying extratropical troughs, which contain a large phase speed component (over the tracking interval, the observed WVWV displacement is not a good representation of the instantaneous flow). Fortunately, the above conditions represent relatively rare situations when considering full-disk (roughly $65^{\circ}$ radius from satellite subpoint) coverage, and experience shows good coverage is attainable over the large majority of meteorological conditions.

In summary, the coverage and quality of WVWV will vary depending on atmospheric factors such as moisture availability and structure, vertical shear, and instrument characteristics such as detector precision and resolution. The next section will evaluate the accuracy of WVWV generated by the CIMSS tracking system through comparisons with rawinsonde observations.

\section{WVWV verification}

The evaluation of WVWV must take into account both qualitative and quantitative measures. Certainly, to observationalists and operational forecasters, the complementary nature of the WVWV to clouddrift winds in cloudy regions is an important enhancement to the qualitative analysis of the uppertropospheric wind field. For example, it was shown in the last section how the WVWV provided information on the environment of Tropical Storm Pablo that may have been useful in the intensity forecast. Another good example is the WVWV fields being derived routinely from $G M S$ - 5 in a demonstration mode by CIMSS for the Joint Typhoon Warning Center (JTWC) at Guam. These data are being integrated into the JTWC subjective upper-level analyses, and feedback has been very positive (further details in section 7 ).

Quantitative assessment of the WVWV product is possible from statistical analyses and impact on NWP. The latter will be addressed in the next section. A traditional method of validation is matching observations with collocated rawinsondes. These statistics can provide a fixed measure of product quality over time and can be employed in determining observation weight in objective data assimilation.

Validation statistics for WVWV from the GOES-8 6.7- $\mu \mathrm{m}$ channel are shown in Table 2 . These WVWV were produced by NESDIS using the CIMSS algorithm during the months of October-December 1995 [at the time this article was submitted, the NESDIS 
WVWV were being produced in a quasioperational mode and evaluated by NCEP-EMC (Environmental Modeling Center) for consideration as input to NCEP operational models]. Table 2 shows that the overall quality controlled WVWV root-mean-square errors (rmse) during this period were $7.47 \mathrm{~m} \mathrm{~s}^{-1}$, with a bias of $-0.26 \mathrm{~m} \mathrm{~s}^{-1}$. These rmse inherently include errors in rawinsonde measurement and reporting, which are typically around $3 \mathrm{~m} \mathrm{~s}^{-1}$ (Hoehne 1980). The statistics are further stratified by vector height and location. Generally, the best results are achieved in the upper troposphere (above $400 \mathrm{mb}$ ) and in the Tropics, for reasons related to characteristics discussed in the previous section.

It is insightful to compare the statistics of WVWV with operational cloud drift winds from $G O E S-8$ produced by NESDIS over the same period (Table 3 ). Only the high- and midlevel stratifications can be evaluated, since there are no low-level (below $700 \mathrm{mb}$ ) WVWV. Comparatively, the quality of high-level WVWV match well with the operational cloud drift winds but is slightly inferior at midlevels (below $400 \mathrm{mb}$ ). However, the coverage and information in the midlevels is almost twice that of the cloud-drift winds.

The statistics presented in Table 2 are a reflection of WVWV produced with a rather stringent internal quality control that is being applied in the postprocessing stage. NESDIS and operational considerations demand this approach. However, research at CIMSS is suggesting an alternate processing strategy aimed at relaxing the QC constraints slightly in favor of retaining information in key meteorological areas (discussed in section 3 ). The result of this approach yields vector rmse that are slightly higher. However, the tradeoff can be dramatic in terms of retaining vector coverage and information in regions of model guess deficiencies. For example, as shown earlier, the application of WVWV data to the analyses of tropical cyclone wind fields can yield significant information on the upper-level environmental circulations affecting storm motion (Velden 1996; Franklin et al. 1996).

For purposes of data assimilation, the WVWV accuracies are related to vector wind speed in Fig. 8. Generally speaking, vector rmse increase steadily with wind speed. Part of this may be attributed to increased errors in rawinsonde measurements at higher wind speeds (SRCC 1981). However, if the WVWV rmse values are considered as a percent of vector speed, the accuracy actually increases in higher wind regimes.

It was mentioned in the previous section that WVWV are also possible from GOES sounder channels measuring radiances at central wavelengths of 7.0 and $7.3 \mu \mathrm{m}$, which allow sensing in cloud-free regions in the midtroposphere (Fig. 3). Statistical evaluations done at CIMSS show these vector rmse to be comparable to the $6.7-\mu \mathrm{m}$ WVWV. Figures 9 and 10 illustrate the vector rmse relative to wind speed for the 7.0and 7.3- $\mu \mathrm{m}$ WVWV, respectively. The magnitude and characteristic increase in rmse with respect to vector speed is similar to that found with the 6.7- $\mu \mathrm{m}$ WVWV. Comparisons in higher wind speed regimes are more limited due to the nature of the sounder WVWV sensing at relatively lower tropospheric levels (and wind speeds). In the midlevels, the lack of good moisture signatures or multiple moist layers within the energy contribution function layer is more common and poses a greater challenge to the retrieval of accurate tracers and attendant height assignments. Preliminary statistical analyses and NWP results from NCEP-EMC are indicating a 1-2 $\mathrm{m} \mathrm{s}^{-1}$ slow speed bias in the $7.3-\mu \mathrm{m}$ WVWV in the lowest levels. This is being investigated. 
TABLE 2. Rawinsonde verification statistics for GOES-8 $6.7-\mu \mathrm{m}$ water vapor winds during October-December 1995. The collocation match radius does not exceed 2 degrees. Root-meansquare vector error (rmse), standard deviation of the vector difference (stdv), mean speed difference (bias), rawinsonde speed (spd) (all $\mathrm{m} \mathrm{s}^{-1}$ ), and number of collocated matches (num) are shown. High represents WVWV between 100 and $399 \mathrm{mb}$, and mid is $400-699 \mathrm{mb}$.

\begin{tabular}{|c|c|c|c|c|}
\hline & $\begin{array}{c}\text { All } \\
\text { regions }\end{array}$ & $\begin{array}{c}\text { NH } \\
\text { extratopics }\end{array}$ & Tropics & $\begin{array}{c}\text { SH } \\
\text { extratropies }\end{array}$ \\
\hline \multicolumn{5}{|c|}{ All levels } \\
\hline Rmse & 7.47 & 7.97 & 5.61 & 8.02 \\
\hline Stdv & 4.45 & 4.71 & 3.08 & 4.77 \\
\hline Bias & -0.26 & -0.32 & -0.16 & 0.78 \\
\hline Spd & 23.04 & 26.87 & 10.69 & 18.97 \\
\hline Num & 9337 & 7030 & 2118 & 194 \\
\hline \multicolumn{5}{|l|}{ High } \\
\hline Rmse & 7.45 & 7.95 & 5.62 & 8.37 \\
\hline Stdv & 4.43 & 4.71 & 3.09 & 4.92 \\
\hline Bias & -0.13 & -0.14 & -0.17 & 0.74 \\
\hline Spd & 23.38 & 27.70 & 10.75 & 19.72 \\
\hline Num & 8378 & 6151 & 2067 & 165 \\
\hline \multicolumn{5}{|l|}{ Mid } \\
\hline Rmse & 7.93 & 8.12 & 5.57 & 5.69 \\
\hline Stdv & 4.60 & 4.70 & 2.63 & 3.32 \\
\hline Bias & -1.41 & -1.58 & 0.18 & 0.97 \\
\hline Spd & 20.15 & 21.02 & 8.15 & 14.66 \\
\hline Num & 959 & 879 & 51 & 29 \\
\hline
\end{tabular}

CIMSS is routinely producing WVWV twice a day from $G M S-5$ for analysis by the JTWC. The processing strategy is geared to a high-density product covering the western North Pacific Ocean, with adjusted QC constraints as outlined in section 3. An initial evaluation of these observations versus rawinsonde data is yielding vector quality on par with what is
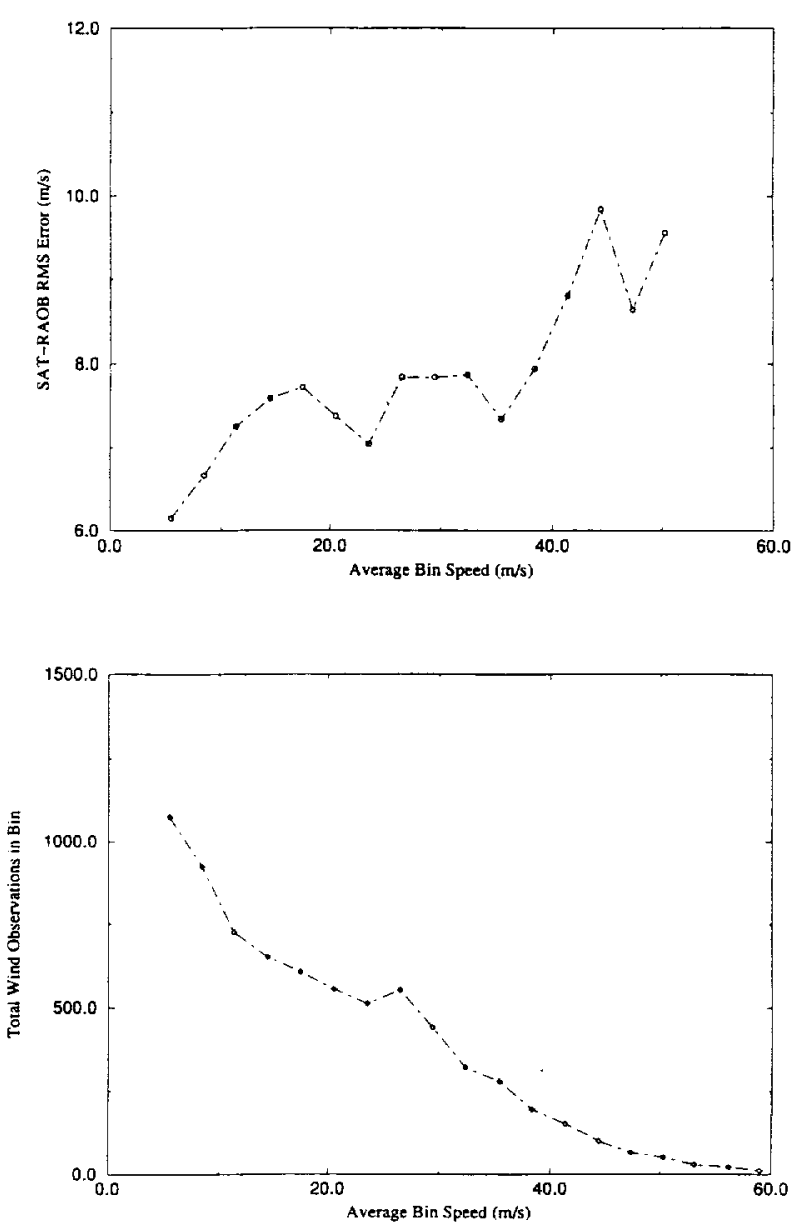

FIG. 8. (Top): Accuracy (vector rms difference) of $6.7-\mu \mathrm{m}$ $G O E S-8$ water vapor wind vectors produced quasi-operationally by NESDIS compared to collocated rawinsondes over the United States with respect to vector wind speed ( $3 \mathrm{~m} \mathrm{~s}^{-1}$ bins). (Bottom): Number of wind-rawinsonde matches per wind speed bin. The matches were collected during the period of October-December 1995.

being observed with GOES WVWV being produced at CIMSS: rmse of $7.0 \mathrm{~m} \mathrm{~s}^{-1}$, with a bias of $-0.5 \mathrm{~m} \mathrm{~s}^{-1}$. The GMS-5 WVWV accuracies relative to vector speed are shown in Fig. 11. The functional relationship is quite similar to that for GOES-8 illustrated in Figs. 8-10. The Japanese Meteorological Agency (JMA) is also experimenting with automated techniques to produce WVWV from the GMS-5. At the time of writing, this product is being evaluated for operational use at the JMA.

\section{NWP data sensitivity experiments}

The importance of observations is often measured in terms of the impact on objective analyses and nu- 


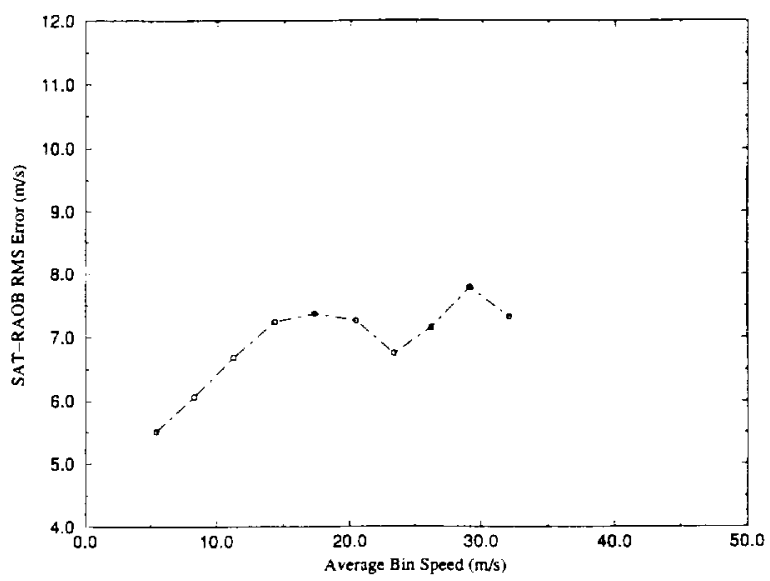

TABLF 3. Rawinsonde verification statistics for GOES-8 IR cloud-drift winds during October-December 1995 . The collocation match radius does not exceed 2 degrees. Root-mean-square vector error (rmse), standard deviation of the vector difference (stdv), mean speed difference (bias), rawinsonde speed (spd) (all $\mathrm{m} \mathrm{s}^{-1}$ ), and number of collocated matches (num) are shown. High represents winds between 100 and $399 \mathrm{mb}$, mid is $400-699 \mathrm{mb}$, and low is $700-950 \mathrm{mb}$.

\begin{tabular}{|c|c|c|c|c|}
\hline & $\begin{array}{c}\text { All } \\
\text { regions }\end{array}$ & $\begin{array}{c}\text { NH } \\
\text { extratopics }\end{array}$ & Tropics & $\begin{array}{c}\text { SH } \\
\text { extratropics }\end{array}$ \\
\hline \multicolumn{5}{|c|}{ All levels } \\
\hline Rmse & 7.07 & 7.50 & 5.47 & 7.54 \\
\hline Stdv & 4.26 & 4.54 & 2.98 & 4.85 \\
\hline Bias & -0.40 & -0.41 & -0.46 & 0.36 \\
\hline Spd & 19.20 & 22.31 & 10.48 & 16.46 \\
\hline Num & 2823 & 2039 & 703 & 81 \\
\hline \multicolumn{5}{|l|}{ High } \\
\hline Rmse & 7.44 & 7.88 & 5.93 & 8.32 \\
\hline Stdv & 4.41 & 4.72 & 3.19 & 5.12 \\
\hline Bias & -0.43 & -0.26 & -0.97 & 0.54 \\
\hline Spd & 22.59 & 26.81 & 11.81 & 20.80 \\
\hline Num & 1917 & 1346 & 517 & 54 \\
\hline \multicolumn{5}{|l|}{ Mid } \\
\hline Rmse & 6.34 & 6.64 & 4.40 & 4.22 \\
\hline Stdy & 3.72 & 3.90 & 2.04 & 2.40 \\
\hline Bias & -0.52 & -0.70 & 0.61 & -0.70 \\
\hline Spd & 13.94 & 15.18 & 6.43 & 12.18 \\
\hline Num & 554 & 469 & 75 & 10 \\
\hline \multicolumn{5}{|l|}{ Low } \\
\hline Rmse & 5.97 & 6.84 & 3.33 & 6.50 \\
\hline Stdv & 3.95 & 4.52 & 1.46 & 4.56 \\
\hline Bias & -0.04 & -0.66 & 1.15 & 0.39 \\
\hline Spd & 8.96 & 10.23 & 6.97 & 5.18 \\
\hline Num & 352 & 224 & 111 & 17 \\
\hline
\end{tabular}

FIG. 9. Same as Fig. 8 except for WVWV produced by CIMSS from the $7.0-\mu \mathrm{m}$ channel.

merical prognoses. Previous studies have provided a glimpse into the impact of WVWV data on NWP. Despite a nonoptimized assimilation process, the data were found to have a positive impact on regional forecasts utilizing a limited-area prediction system (Velden 1996). However, the impact was modest. The WVWV were also assimilated into a low-resolution (T62) version of the NCEP global spectral model for a period of a month in 1994. The results were not statistically significant, but virtually all parameters examined exhibited a small positive impact (Velden 1996). The strongest positive impact was noted in the Tropics, in particular, the rotational component of the wind.

It was shown in Velden et al. (1992) that the inclusion of high-density satellite-derived wind information into the analyses of tropical cyclone environmental wind fields can effectively reduce the error of objective track forecasts. This particular study 

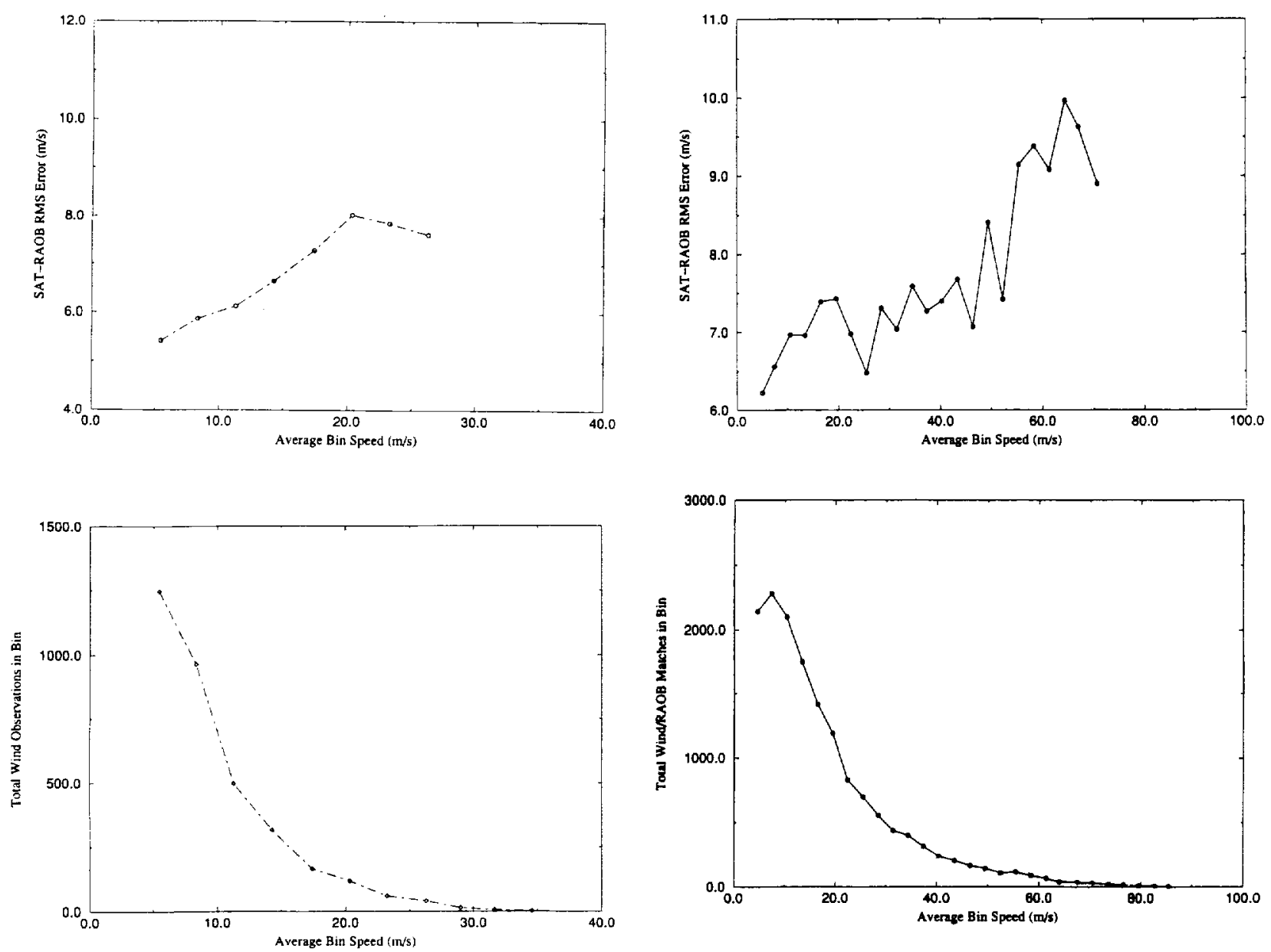

FIG. 10. Same as Fig. 8 except for WVWV produced by CIMSS from the 7.3- $\mu \mathrm{m}$ channel.

did not evaluate WVWV; however, a later investigation tested the sensitivity of GOES WVWV data on a spectral barotropic numerical hurricane track forecast model (Velden 1996). It was found that small improvements resulted in numerical track forecasts with the inclusion of the WVWV observations, despite the limitations of the analysis and forecast system employed.

A natural extension to these previous track forecast studies is to assimilate the data into state-of-theart, primitive-equation analysis and prediction systems in order to take full advantage of the satellite information and maximize the potential impact. Prediction systems developed at the Naval Research Laboratory (NRL) for operational use at the navy's Fleet Numerical Meteorology and Oceanography Center (FNMOC) and from the Geophysical Fluid Dynamics Lab (GFDL) have shown significant skill in hurricane track forecasting. The NRL-FNMOC model (NOGAPS) is a global assimilation system that

contains special tropical cyclone bogusing procedures (Goerss and Jeffries 1994). The GFDL model is a limited-area, multinested system that employs NCEP global fields as background information (Kurihara et al. 1995). Both systems were top performers in terms of objective hurricane track predictions during the prolific 1995 Atlantic season.

These two prediction systems were employed to evaluate WVWV datasets prepared by CIMSS during the 1995 tropical cyclone season (Northern Hemisphere). Data were assimilated into NOGAPS for Atlantic Tropical Storm Chantal and western North Pacific Typhoons Ward and Angela. The Chantal datasets included winds derived at 12 -h intervals from GOES-8 infrared (IR) and visible (VIS) imagery, and WVWV from the aforementioned three GOES- $8 \mathrm{WV}$ bands. The WVWV outnumber the IR-VIS winds by a factor of 6 and hence dominate the sample. The Ward and Angela GMS-5 WVWV datasets were pro- 
cessed at 6-h intervals. The GFDL model was also run on the Chantal case and on Hurricane Gordon (1994) using NCEP operational global analyses as the largescale background fields, which were modified by the assimilated winds. The Gordon datasets contained only GOES-8 6.7- $\mu \mathrm{m}$ WVWV, processed at $12-\mathrm{h}$ intervals and were derived during the period when Gordon was upstream (south) of the dense U.S. upperair observing system (Avila and Rappaport 1996).

The results of the two-system prediction trials are given in Table 4. Track forecast errors were computed by using the observed track as verification and calculating the distance between the observed and forecast locations. Mean forecast error improvement (expressed as an overall percentage improvement over control runs that did not include the satellite data) and the number of improved forecasts relative to control runs are evaluated for each storm. Results are presented for track predictions at 24,48 , and $72 \mathrm{~h}$. Generally speaking, the data impacts are positive, including a significant reduction in forecast errors for the NOGAPS Chantal case ( $31 \%$ at $48 \mathrm{~h}$ ). An exception is Supertyphoon Ward, which exhibited slight negative data impacts early on. In this case, the definition of the broad upper-level outflow picked up by the WVWV was detrimental to the model steering. Overall, considering all cases from both model trials, assimilation of the wind information results in a reduction of mean forecast errors by $6 \%-13 \%$ over the 24-72-h forecast period (all significant at the $95 \%$ level). The number of improved forecasts versus control is also quite encouraging.

It is informative to dissect the NOGAPS performance in the Chantal case since the most significant results were achieved there (all forecasts were improved at 48 and $72 \mathrm{~h}$ ) and its datasets involved multispectral winds. Table 5 presents the statistics for the control (CON) and satellite data enhanced (CON + SAT) trials. The results are also compared to the climate-persistence (CLIPER) model. The reduction of mean forecast errors for the CON + SAT are considerable when compared to both the CON and CLIPER. The forecast error reduction is significant at $>92 \%$ level for all forecast periods.

A satellite component analysis of the Chantal case is given in Table 6. In this set of trials, NOGAPS forecasts were produced from analyses that excluded each individual component of the multispectral dataset in order to glean information on the impact of each data type. The results of the component analysis in this case reveal 1) the most significant contributor to error re-
TABLE 4. Numerical weather prediction data (WVWV) impact results for selected tropical cyclones. Mean track forecast errors (MFE) are evaluated and the results are expressed as a percentage improvement over the control (without WVWV) forecasts. GFDL is the Geophysical Fluids Dynamics Laboratory hurricane model and NOGAPS is the Navy Operational Global Atmospheric Prediction System.

\begin{tabular}{|c|c|c|c|}
\hline & \multicolumn{3}{|c|}{ Forecast interval (h) } \\
\hline & 24 & 48 & 72 \\
\hline \multicolumn{4}{|l|}{ Hurricane Gordon (1994) } \\
\hline GFDL & 15 & 19 & 5 \\
\hline No. of cases (no. improved) & $6(5)$ & $6(5)$ & $6(4)$ \\
\hline \multicolumn{4}{|l|}{ Supertyphoon Ward (1995) } \\
\hline NOGAPS & -6 & -4 & 0 \\
\hline No. of cases (no. improved) & $9(3)$ & $7(2)$ & $5(2)$ \\
\hline \multicolumn{4}{|l|}{ Supertyphoon Angela (1995) } \\
\hline NOGAPS & 13 & 6 & 14 \\
\hline No. of cases (no. improved) & $7(5)$ & $7(6)$ & $7(4)$ \\
\hline \multicolumn{4}{|c|}{ Tropical Storm Chantal (1995) } \\
\hline GFDL & -9 & 12 & 12 \\
\hline No. of cases (no. improved) & $10(4)$ & $8(6)$ & $6(5)$ \\
\hline \multicolumn{4}{|c|}{ Tropical Storm Chantal (1995) } \\
\hline NOGAPS & 20 & 31 & 20 \\
\hline No. of cases (no. improved) & $10(8)$ & $8(8)$ & $6(6)$ \\
\hline $\begin{array}{l}\text { Overall MFE } \\
\text { improvement }(\%)\end{array}$ & 6 & 13 & 10 \\
\hline No. of cases (no. improved) & $42(25)$ & $36(27)$ & $30(21)$ \\
\hline $\begin{array}{l}\text { Overall MFE }(\mathrm{nm}) \\
\text { for control }\end{array}$ & 94 & 144 & 197 \\
\hline $\begin{array}{l}\text { Overall MFE (nm) } \\
\text { for control + sat }\end{array}$ & 88 & 125 & 177 \\
\hline
\end{tabular}

duction is the WVWV employed collectively, 2) the GOES sounder WVWV (7.0- and 7.3- $\mu \mathrm{m}$ observations) is an important individual component at $24 \mathrm{~h}$ 
TABLE 5. NOGAPS forecast impact results for Tropical Storm Chantal (1995). CLIPER is the climate persistence model, a standard for assessing skill. Mean forecast errors (MFE) are in nautical miles.

\begin{tabular}{lccc}
\hline & \multicolumn{3}{c}{ Forecast interval (h) } \\
& $\mathbf{2 4}$ & $\mathbf{4 8}$ & $\mathbf{7 2}$ \\
\hline Control (CON) MFE & 100 & 179 & 301 \\
$\begin{array}{l}\text { Control + satwinds MFE } \\
\text { (CON + SAT) }\end{array}$ & 80 & 124 & 242 \\
CLIPER MFE & 112 & 210 & 358 \\
\hline $\begin{array}{l}\text { \% improvement } \\
\text { (CON + SAT over CON) }\end{array}$ & 20 & 31 & 20 \\
$\begin{array}{l}\text { \% improvement } \\
\text { (CON + SAT over CLIPER) }\end{array}$ & 29 & 41 & 33 \\
\hline \# forecasts & & & \\
\hline $\begin{array}{l}\text { \# improved forecasts } \\
\text { (CON + SAT over CON) }\end{array}$ & 8 & 8 & 6 \\
\hline $\begin{array}{l}\text { T-test significance (\%) } \\
\text { (CON + SAT over CON) }\end{array}$ & 92 & 97 & 93 \\
\hline
\end{tabular}

and is also a significant contributor at $48 \mathrm{~h}, 3$ ) the imager $(6.7 \mu \mathrm{m})$ WVWV contributes significantly to the medium- and longer-range forecasts, 4) the highdensity IR cloud-tracked winds are an important component at $24 \mathrm{~h}$, and 5) the low-level visible winds do not appreciably contribute to the forecast improvement. This last point is likely a result of the fact that the VIS winds covered a very limited domain surrounding the storm that mainly just included the outer vortex region and were difficult to obtain in regions covered by the storm cirrus canopy. In addition, the NOGAPS contains a vortex bogusing scheme (Goerss and Jeffries 1994) that may have dampened the effects of these data.

The revelations from the component analysis support intuitive reasoning. The IR winds are most abundant in the storm region (cirrus canopy) and therefore contribute to short-term forecast improvements. The sounder WVWV provide information in the midlevels, closest to the storm steering current, and therefore have a considerable impact on the model track forecasts. However, the limited viewing domain of the GOES- 8 sounder confines the WVWV coverage and
TABLE 6. NOGAPS sensitivity study of the impact of individual GOES-8 wind components in terms of the reduction on MFE for the Tropical Storm Chantal case. SNDRH2O is the combined sounder WVWV, IMGRH2O is the 6.7- $\mu \mathrm{m}$ WVWV, ALLH2O is the combined imager and sounder WVWV, VIS is visible cloudtracked winds, and IR is infrared cloud-tracked winds. Each component is subtracted from the collective dataset and the subsequent NOGAPS forecast results are shown. Mean forecast errors are in nautical miles.

\begin{tabular}{lccc}
\hline & \multicolumn{4}{c}{ Forecast interval (h) } \\
& $\mathbf{2 4}$ & $\mathbf{4 8}$ & $\mathbf{7 2}$ \\
\hline Control (CON) & 100 & 179 & 301 \\
Control + satwinds & 80 & 124 & 242 \\
Control + satwinds - SNDRH2O & 94 & 148 & 246 \\
Control + satwinds - MMGRH20 & 82 & 151 & 280 \\
Control + satwinds - ALLH2O & 97 & 156 & 281 \\
Control + satwinds - VIS & 82 & 133 & 239 \\
Control + satwinds - IR & 90 & 126 & 236 \\
\hline
\end{tabular}

restricts the impact to the near environment in this case, hence, the shorter-term forecasts. The broad spatial coverage of the imager WVWV allows definition of adjacent synoptic features and results in a contribution to forecast improvements at the medium and longer ranges (48-72 h). However, the primary message from this component analysis is that, in this particular case, the NOGAPS assimilation process benefits from the three-dimensional nature of the multichannel observations, and the optimum results are achieved when the multispectral data are considered collectively.

Additional model data impact experiments are being performed at NRL. Also, recent changes in NCEP analysis and assimilation strategies (including the direct assimilation of satellite radiances) are leading to improved large-scale analyses. The ability of the WVWV observations to significantly contribute to the reduction in track forecast errors resulting from the GFDL model initialized with this new analysis system is being investigated. In the meantime, WVWV datasets are being produced in real time by CIMSS and routinely assimilated by FNMOC into the operational NOGAPS. These datasets are also being used in qualitative analyses by the NCEP Tropical Predic- 
tion Center in Miami and the U.S. military`s JTWC at Guam.

\section{Further applications}

The capability exists for current geostationary satellites to provide nearly global WVWV coverage (excluding polar regions). As discussed in the previous section, the operational utility of these data includes assimilation into global prediction systems, which should benefit from the increased information. The WVWV data can also contribute to research studies and operational problems involving regional analyses and local meteorological phenomena, particularly those occurring over marine areas. Applications to studies involving interannual scales are also possible. This section will briefly present a few examples and discuss other potential applications.

\section{a. Midlatitude oceanic analyses}

Forecasters have relied on subjective interpretation of water vapor imagery as an aid to analyzing meteorological conditions over oceanic regions (Weldon and Holmes 1991; Velden 1987). The WVWV information can supplement this in two ways: quantifying the motion of features into actual wind speeds and associating pressure heights with these features. Proper diagnosis of developing meteorological situations will benefit from this additional quantitative information.

The eastern North Pacific is a region lacking in conventional observations, yet important to forecasts affecting U.S. interests. An example of WVWV coverage attainable from $G O E S-96.7-\mu \mathrm{m}$ observations is shown in Fig. 12. In this case, from 29 January 1996, a large evolving oceanic cyclone is present. The representation of upper-level flow around this system is well captured in the WVWV data. Case studies have shown that incorporation of these observations into

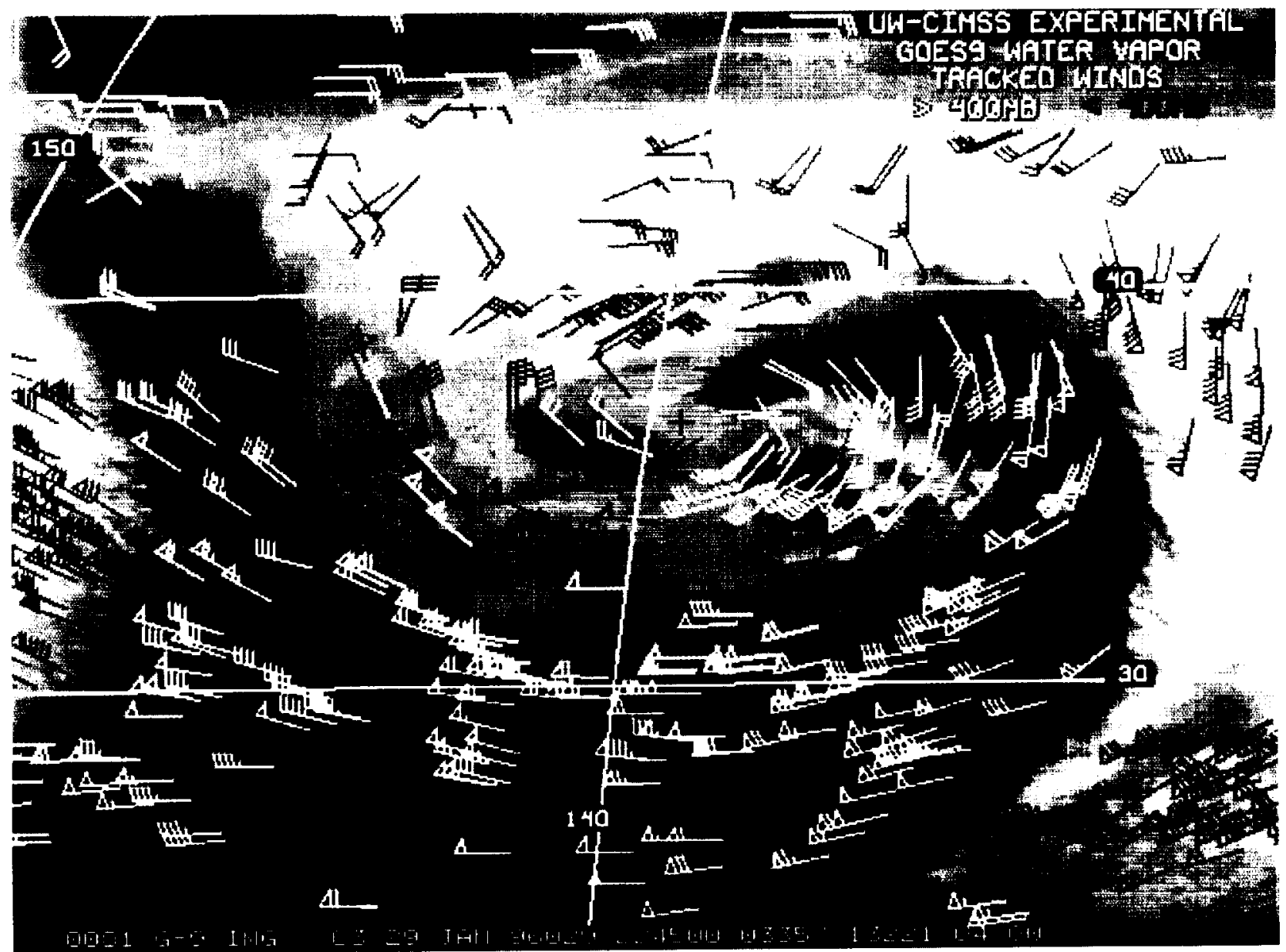

FIG. 12. Example of WVWV from GOES-9 covering a strong extratropical cyclone in the eastern North Pacific on 29 January 1996. Blue (yellow) vectors $(\mathrm{kt}$ ) indicate winds with assigned heights above (below) $400 \mathrm{mb}$. 
regional objective analyses can lead to improved medium-range forecasts (Velden et al. 1994; Velden 1996). The potential addition of multilevel WVWV information from GOES-9 sounder bands over this region is particularly intriguing (Fig. 13) and is the focus of model impact studies under way.

Improving the understanding of developing extratropical oceanic cyclones has been the goal of several

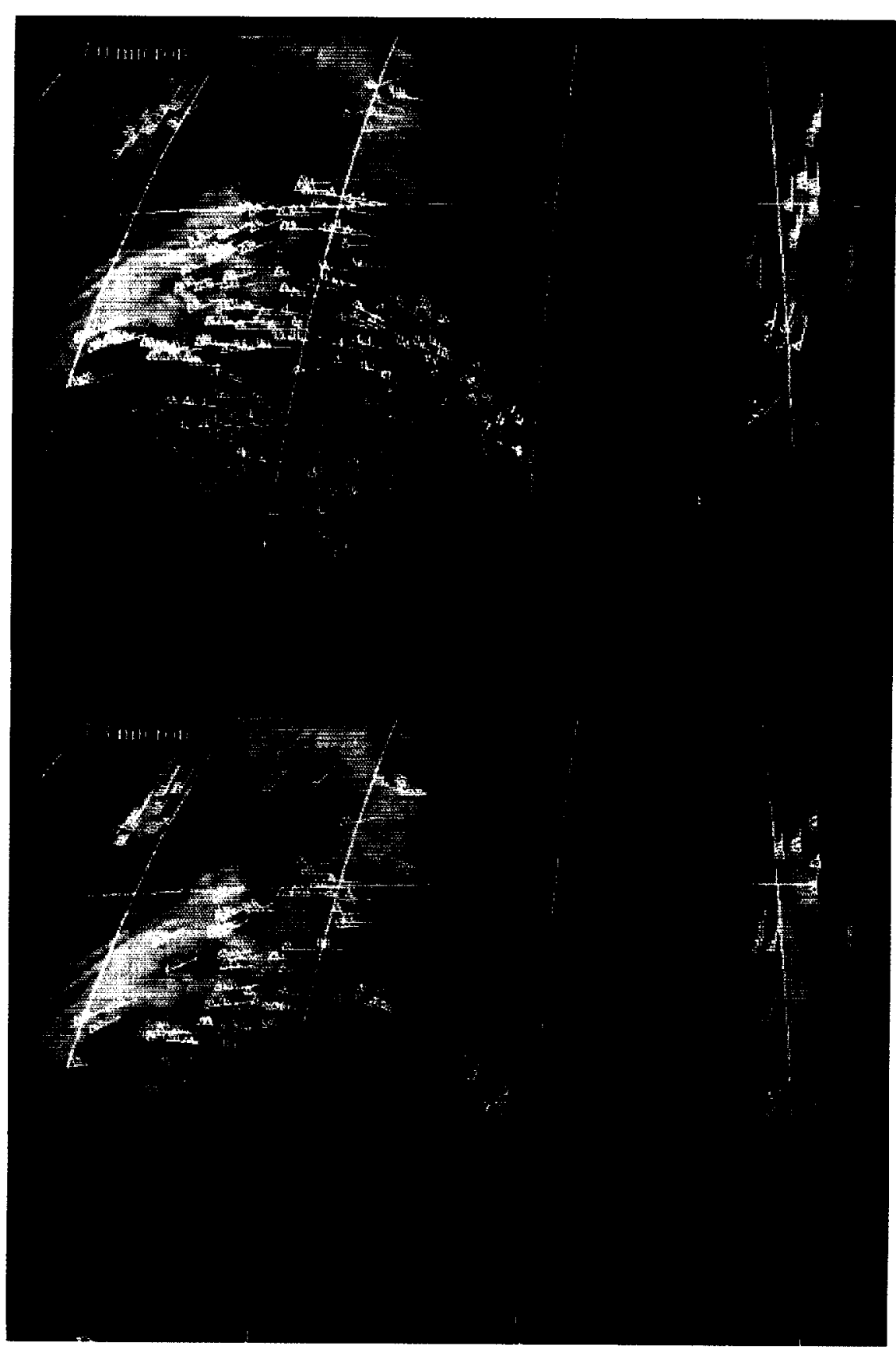

Fig. 13. Example of GOES-9 WVWV derived from sounder bands at $7.0 \mu \mathrm{m}$ (top) and $7.3 \mu \mathrm{m}$ (bottom) on 19 April 1996. Yellow (beige) vectors ( $\mathrm{kt}$ ) indicate winds with assigned heights above (below) $400 \mathrm{mb}$. The winds are plotted over their respective channels. meteorological field experiments over the past decade. The latest of these, Fronts and Atlantic Storm Tracks Experiment (FASTEX), will occur in 1997 (M. Shapiro 1996, personal communication). It is planned that the intensive observing periods will include high-density WVWV from GOES-8 and Meteosat-5. Integration of these data with in situ aircraft observations, dropsondes, ship rawinsondes, and satellite radiances will hopefully lead to an advance in the scientific understanding necessary to enable detailed diagnosis and prediction of the life cycles of North Atlantic storms.

\section{b. Tropical cyclones}

WVWV from GMS-5 are being routinely processed at CIMSS, delivered to the JTWC at Guam, and integrated into their subjective upper-level analyses. Figure 14 illustrates an example of the typical coverage achieved during this demonstrational support in 1995. Analysts at the JTWC indicate that the data are useful in depicting upper-level features and their evolution, which can play a role in tropical cyclone formation and motion. Particularly in the vicinity of typhoons, the data depict upper-level structure and features not captured in the corresponding global analysis. This is illustrated in Fig. 15, which presents the WVWV for Typhoons Zack and Angela and the corresponding model guess winds (from the 6-h forecast of the NCEP operational global model, interpolated to WVWV location and pressure). This is an area lacking in conventional upper-air observations. Clearly, there are regions in the outflow pattern that are represented very differently. The impact of the WVWV winds on numerical forecasts of Angela's track was presented in the previous section. 


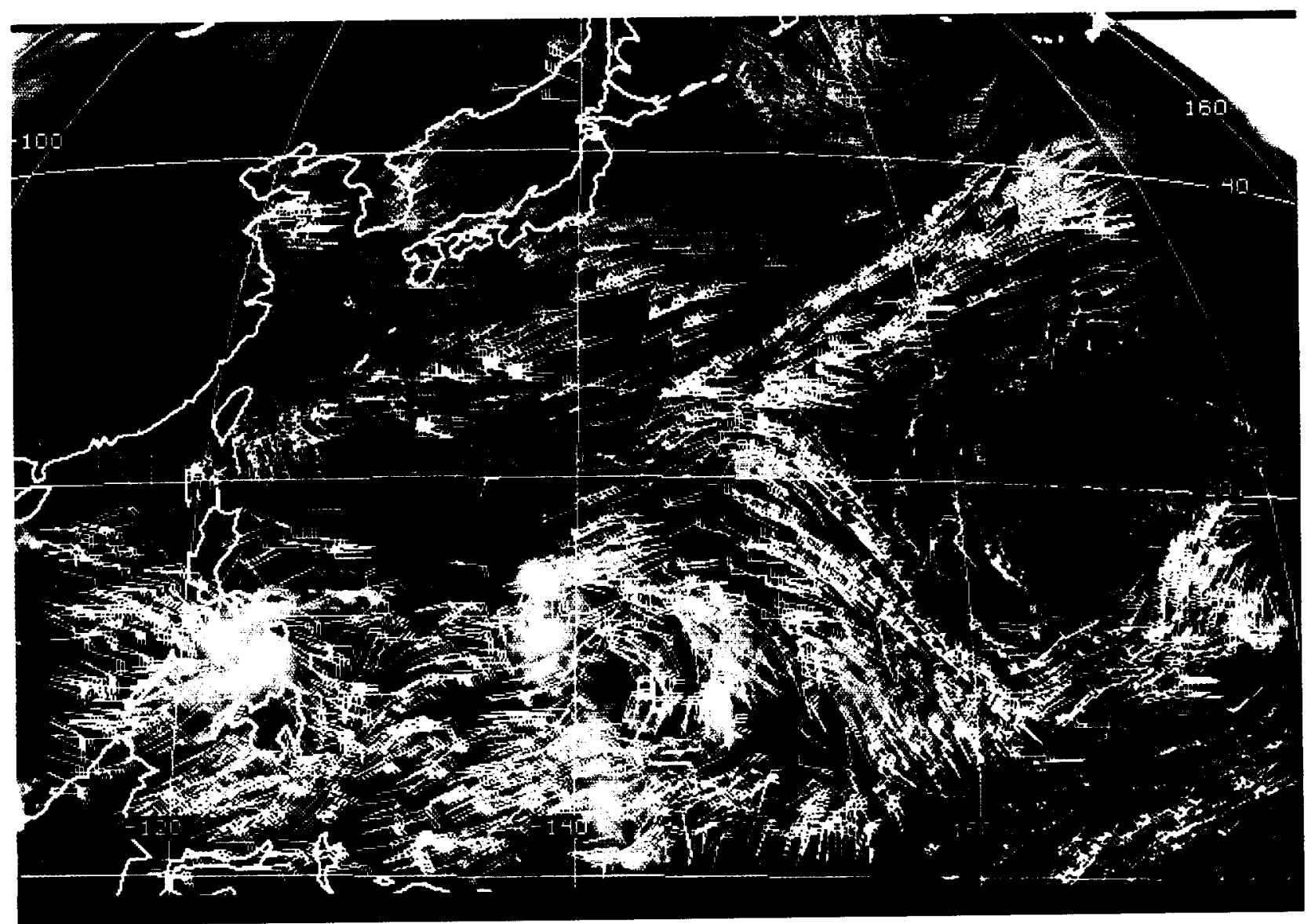

FIG. 14. Example of WVWV routinely produced for JTWC analyses from GMS-5 during Typhoons Zack (125 E) and Angela $\left(137^{\circ} \mathrm{E}\right)$ in the western North Pacific on 28 October 1995. Yellow (beige) vectors $(\mathrm{kt})$ indicate winds with assigned heights above (below) $400 \mathrm{mb}$.

The 1995 Atlantic hurricane season offered many opportunities to assess the GOES-8 WVWV product. Tropical Storm Pablo was a good example of qualitative applications (see earlier discussion). Hurricane Erin presented another fine example. On 31 July, Erin was rapidly developing to hurricane status over the southeastern Bahama Islands. The official track forecast indicated a steady west-northwest movement with the likelihood of landfall over southern Florida within $48 \mathrm{~h}$. The actual track was characterized by a brief jog to the north-northwest on 1 August, which resulted in a landfall over central Florida near Vero Beach. The reason for this track perturbation was evident in GOES- 8 water vapor observations. Image sequences indicated a subtle circulation to the northwest of Erin, traveling to the west. Implications are that Erin and this circulation became briefly involved in a binaryvortex interaction. This midlevel environmental circulation, while subtle, is captured in multispectral WVWV on 1 August (Fig. 16). Operationally available data was not sufficient to describe this feature, resulting in analysis deficiencies and numerical guidance that did not indicate the jog in the track to the northwest.

In addition to operational considerations, the WVWV fields are contributing to research analyses and studies aimed at a better understanding of the processes that can affect tropical cyclone motion and intensity. For example, the NOAA Hurricane Research Division (HRD) began synoptic flow experiments in 1982 that involve special aircraft reconnaissance missions to measure storm environmental parameters (Franklin et al. 1996). These missions collect information from omega dropwindsondes below $350 \mathrm{mb}$ (typical flight level). To supplement these datasets above $350 \mathrm{mb}$, CIMSS provides HRD with high-density multispectral GOES wind observations. From these datasets, objective analyses are being used in diagnostic case studies to examine the influence of synoptic-scale fields on changes in vortex intensity and track, with a goal toward improving short-term track forecasting. 


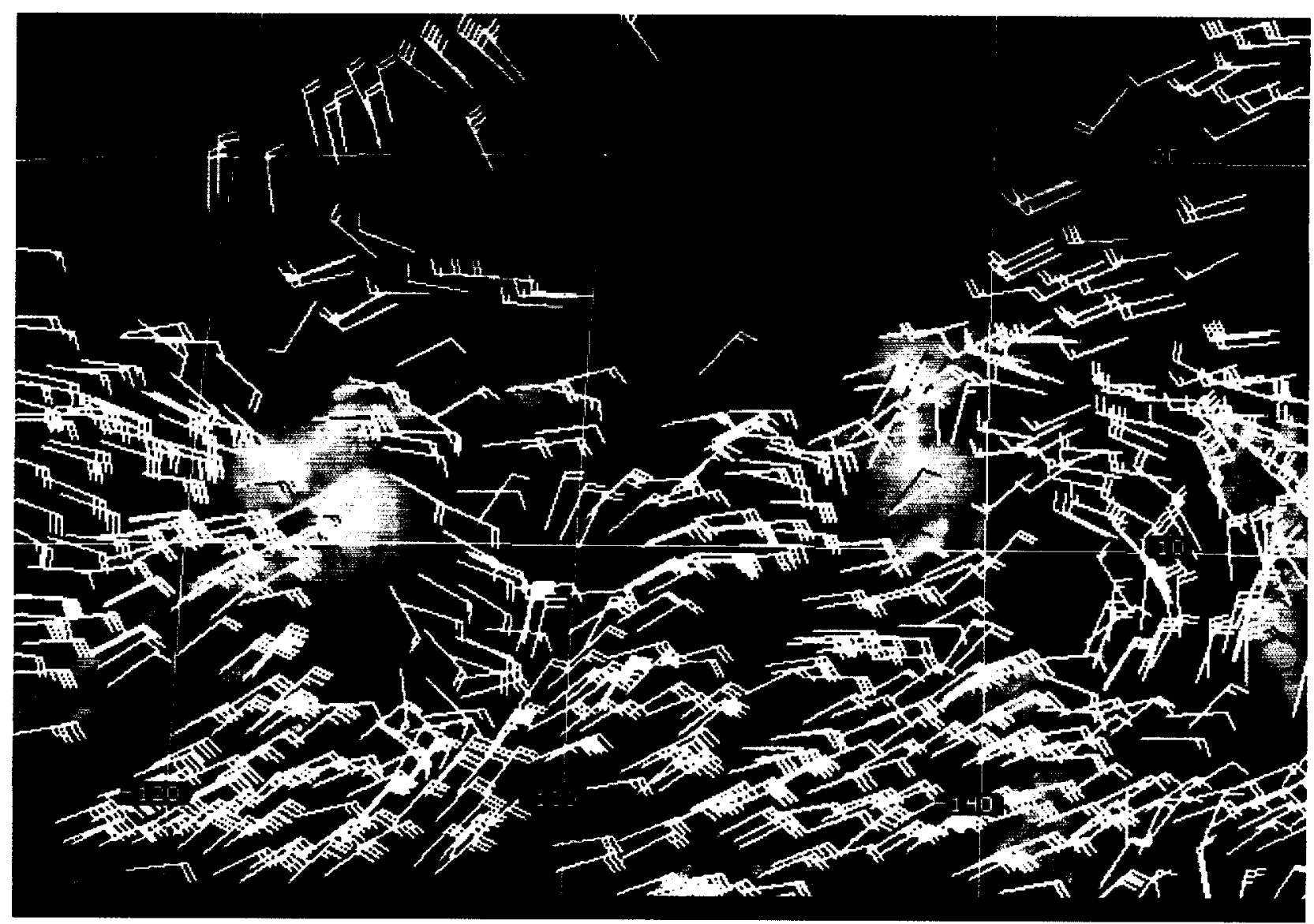

FrG. 15. Plotted WVWV (yellow) and collocated NCEP Aviation model 6-h forecast wind vectors (red) during Typhoons Zack and Angela on 28 October 1995. Only vectors ( $\mathrm{kt}$ ) above $300 \mathrm{mb}$ are shown. Note the differences in the typhoon outflow regimes.

Tropical cyclone environmental steering is commonly associated with a midtropospheric layer-mean flow (Pike 1985; Dong and Neumann 1986; Velden and Leslie 1991). Operational satellite-derived clouddrift winds lack retrievable information in this part of the troposphere. In cloud-free regimes, WVWV can provide midlevel information, particularly if the 7.0and $7.3-\mu \mathrm{m}$ observations are utilized. It was shown in the Tropical Storm Chantal example (previous section) that the ability to retrieve winds in the 400600 -mb layer can be an important contributor to defining the steering flow.

It has long been recognized that the vertical wind shear of the horizontal wind can affect the structure and intensity of tropical cyclones (Gray 1968). More recent studies (Jones 1995; DeMaria 1995) have examined shear in the context that the potential vorticity (PV) associated with the vortex is advected at different rates at different vertical levels. Tilting of PV in the vertical induces circulations that tend to restore the vortex to a vertical orientation and hence main- tain intensity. The ability of the vortex to survive vertical wind shear will depend on the Rossby penetration depth (Hoskins et al. 1985) and the magnitude and profile of the vertical shear. DeMaria and Kaplan (1994) reported on an attempt to quantify vertical shear into a statistical hurricane intensity prediction scheme that has resulted in improvements over climatology persistence. However, the forecasts still explain only about $50 \%$ of the variability of the observed intensity changes. The vertical shear predictor in their scheme is the magnitude of the difference between the 200- and 850-mb wind analyses. These two levels were chosen because most operational satellitederived winds are located around these levels. The scheme may benefit from the multispectral WVWV, which will allow the evaluation of multiple levels.

A real challenge to forecasters is the interaction of tropical cyclones with upper-level troughs, which can frequently affect both the storm motion and intensity. Water vapor image sequences have been used to identify and diagnose these situations (Velden 1987). 


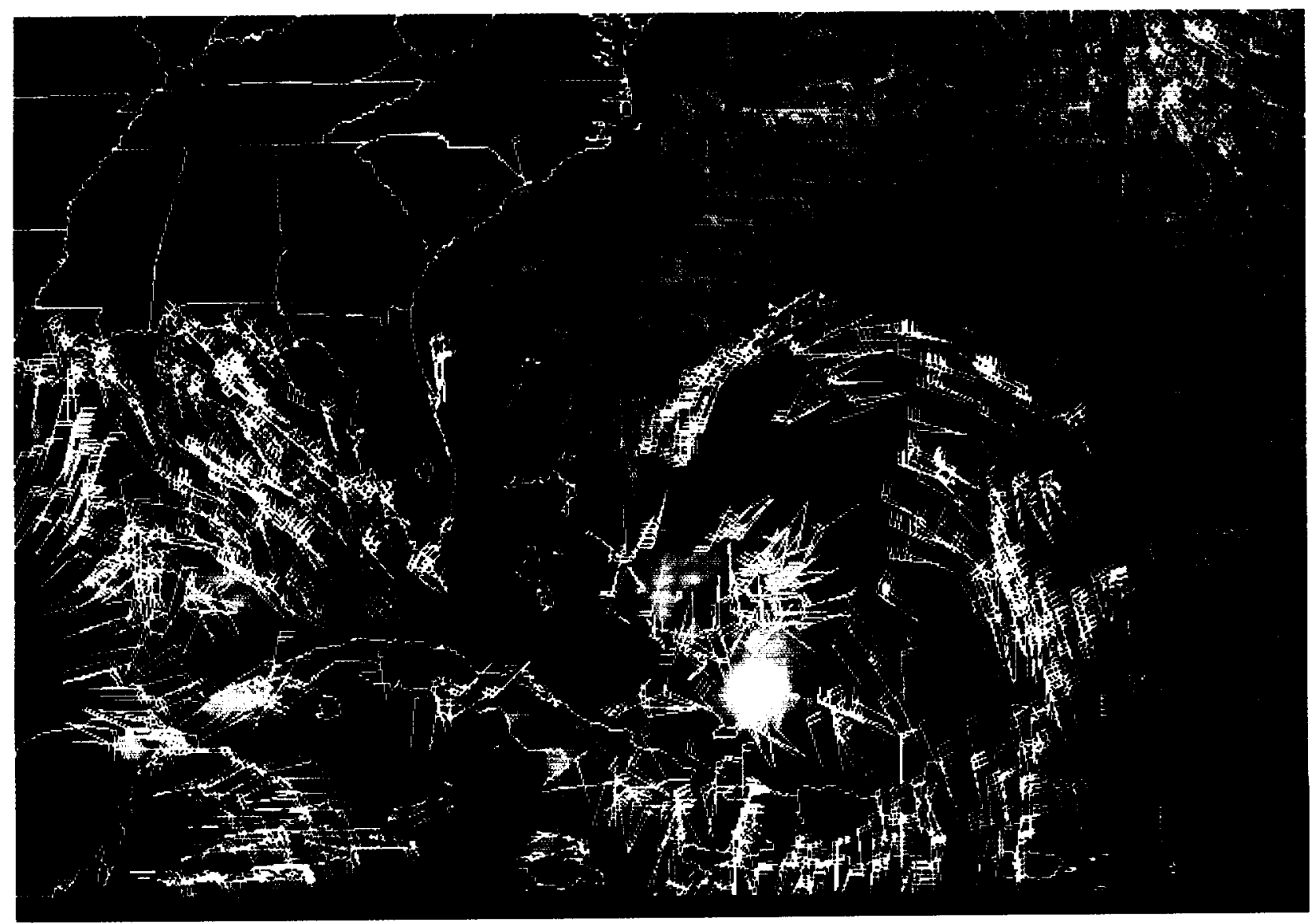

FIG. 16. Plot of multispectral WVWV (kt) derived over marine areas from GOES-8 during Hurricane Erin on 1 August 1995. Yellow (beige) vectors indicate winds with assigned heights above (below) $300 \mathrm{mb}$. Note the circulation to the northwest of Erin that subsequently interacted with the tropical cyclone to alter its track.

Traditional forecast philosophy suggests that these situations purport to be hostile for tropical cyclones, given the presence of strong vertical wind shear commonly found with the troughs. However, recent research based within a PV framework suggests the storm intensity will be modulated by the relative strengths and juxtaposition of the positive PV anomaly associated with the trough, and the negative PV anomaly associated with the outflow anticyclone (Molinari et al. 1995). WVWV can play a key role in describing such interactions, and this is currently being investigated.

\section{c. Seasonal and climate scales}

Water vapor observations from geostationary satellites have been utilized to study short-term climatologies. Specifically, the radiance information from the $6-7.5-\mu \mathrm{m}$ band can be transformed into an upper-tropospheric relative humidity (UTH), which can then be related to atmospheric processes or em- ployed toward model validation (Schmetz and Turpeinen 1988; van de Berg et al. 1991; Soden and Bretherton 1993; Schmetz and van de Berg 1994). Significant differences have been noted between UTH fields derived from satellite observations and those in general circulation models. The natural extension of these studies is to utilize WVWV to infer upper-level moisture transport and to diagnose seasonal or intraannual changes linked to the UTH trends.

Schmetz et al. (1995) describe the congruence of monthly mean fields of UTH and WVWV derived simultaneously from three geostationary satellites. The patterns (their Fig. 5) suggest a physical relationship in which the UTH fields are largely influenced by upper-tropospheric transport. It is qualitatively demonstrated that UTH and upper-tropospheric dynamics are directly observable on a nearly global scale and that the UTH and WVWV fields are desirable contributors to a climate-monitoring system.

Diagnosis of seasonal variations can also benefit 
from the UTH and WVWV observations. For example, the 1994 Atlantic hurricane season was extremely inactive. Although easterly wave disturbances emerging off the western African coast were numerous, only one evolved into a minimal hurricane. Certainly, cooler than normal SSTs in the region played a role, but it is likely that upper-level conditions also contributed to the lack of vertical development and organization of convection associated with the disturbances. To investigate the mean upper-tropospheric conditions present during the 1994 Atlantic hurricane season, UTH and WVWV fields were produced from Meteosat-3 observations nominally twice per day during the months of July-September 1994 and averaged over the 3-month period.

The UTH fields were derived from Meteosat-3 6.5- $\mu \mathrm{m}$ radiance measurements and a concurrent analysis of the state of the atmosphere (NCEP global analysis). The procedure utilized a forward radiative transfer model to compute radiances based on information from the NCEP analysis. The observed radiances were compared with the computed radiances and a log-linear interpolation determined the final UTH value (Schmetz and Turpeinen 1988). The WVWV were also derived from the Meteosat-3 6.5- $\mu \mathrm{m}$ channel in a procedure similar to that described in section 3 . From the 3-month mean WVWV field, a layer-mean divergence was calculated.

The respective mean UTH and divergence fields are shown in Fig. 17. The relatively dry upper troposphere between $12^{\circ}$ and $30^{\circ} \mathrm{N}$, stretching from the African coast to $65^{\circ} \mathrm{W}$, is noteworthy. The layer-mean divergence analysis from the WVWV field indicates this region is characterized by upper-level convergence. Considered together, these fields suggest mean subsidence was present over the region during this period. Such upper-tropospheric conditions may have acted to hinder convection associated with the emerging disturbances, thereby inhibiting further development. This analysis provides supporting evidence that mean conditions over the eastern Atlantic were not favorable for development of westward-traveling disturbances emerging from Africa, which are a primary feature of the general circulation that can provide the initial stimulus for Atlantic hurricane development. A similar study involving analyses from the 1995 Atlantic hurricane season is under way in order to contrast conditions during this prolific hurricane season.

\section{Discussion}

Improved numerical weather analysis and prediction systems are placing increasing demands on observation density and accuracy. Optimized data processing strategies must be developed in combination with increased measurement precision in order

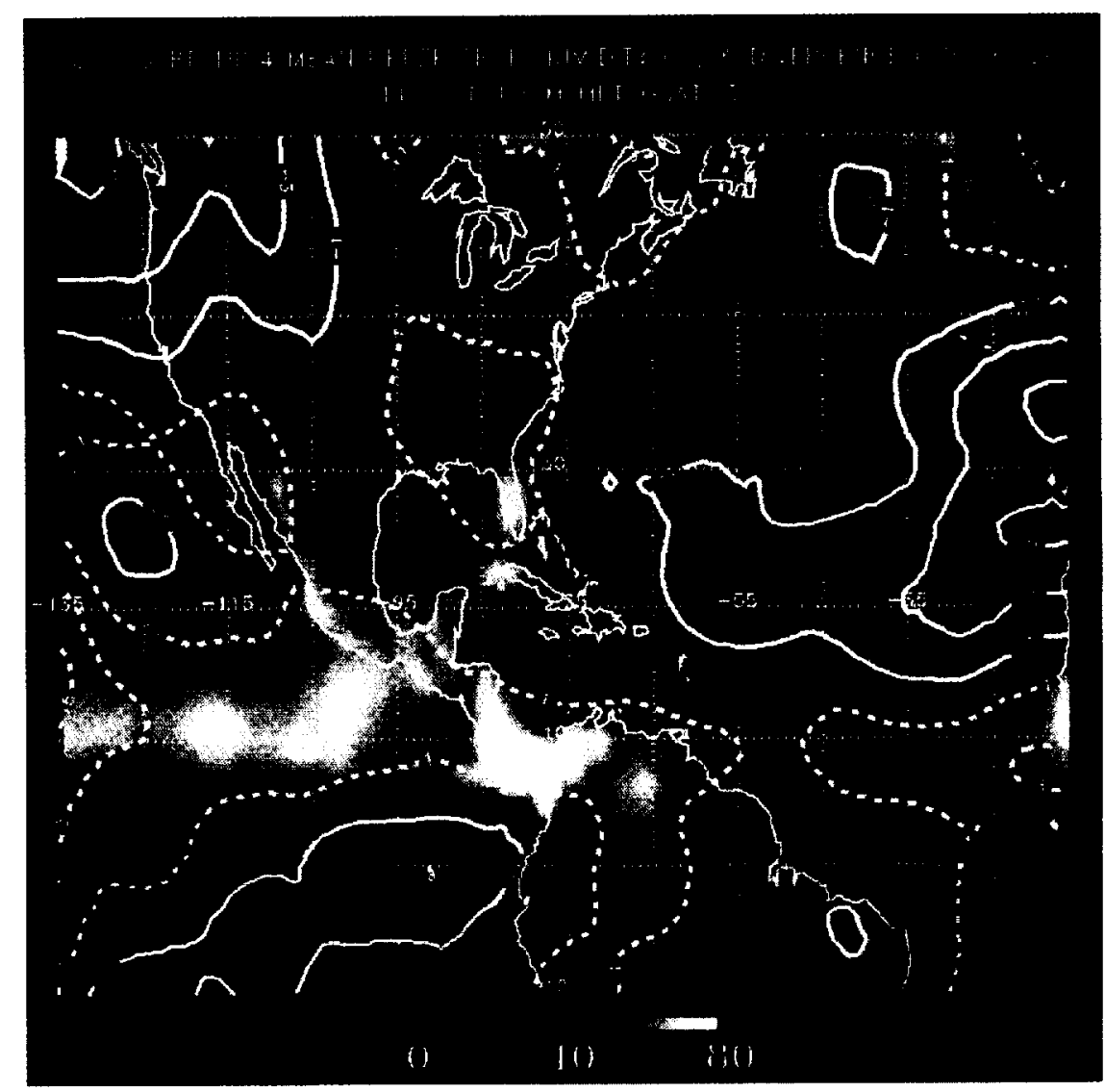

FIG. 17. Composite of 3-month-mean (July-September 1994) upper-tropospheric relative humidity (color code in \%) and divergence (contours, $10^{-6} \mathrm{~s}^{-1}$ ) derived from Meteosat 3 water vapor observations. Solid contours represent convergence. The divergence field was computed from a 3-month-mean WVWV field. 
to meet these requirements. The remote sensing capabilities from geostationary orbit are improving (Menzel and Purdom 1994). In this article, it was shown that initial attempts to optimize the data processing designed to take full advantage of these capabilities can result in spatially coherent and useful measurements of atmospheric motion. In particular, wind vectors derived from water vapor image sequences can complement our existing observing system and provide positive impact on both qualitative and quantitative analyses of the atmospheric state. Further research is necessary to refine the methodologies (e.g., vector height assignments) in order to advance the product quality and analysis impact.

The NWP impact experiments presented in this paper and in previous studies, while limited, are encouraging. Further studies are ongoing to assess the potential impact of the data on improving objective analyses. The WVWV datasets can be reliably produced via automated methodologies at densities to meet varying meteorological requirements, in a time frame commensurate with operational needs. For example, large-scale (full disk) WVWV fields can be processed at reasonable densities for assimilation into global models. These fields can then be augmented with a higher-density product in meteorologically active regions (e.g., tropical cyclones) with an attendant quality control tuned to these active situations. WVWV datasets currently being produced at CIMSS from both GOES and GMS are being incorporated into the navy's operational global assimilation and prediction system (NOGAPS). At the time of this writing, such an approach using GOES WVWV was also being developed and tested by the NESDIS research division for NCEP operational considerations.

A challenge to the NWP community is to continue to evaluate and explore ways to effectively assimilate this data source. Traditionally, three-dimensional assimilation schemes have difficulty in retaining patchy, single-level information (e.g., cloud-drift winds, aircraft reports). Utilized collectively with a similar processing strategy from cloud-tracked winds (Velden et al. 1992; Nieman et al. 1997), the highdensity multispectral WVWV should help retain satellite information in the assimilation process by providing spatially coherent, three-dimensional coverage in the upper troposphere. Clearly, further study is necessary to explore the optimal assimilation of these data into objective analyses (i.e., observational weighting, balancing of the wind information with the mass fields, vertical blending, etc.). The ability of geostationary satellite sensing to provide high temporal interval datasets will also become an advantage to evolving assimilation strategies (e.g., fourdimensional variational schemes).

As illustrated in this article, the WVWV fields cannot only contribute to operational objectives, but can benefit atmospheric research initiatives covering a wide range of scales. Understanding of meteorological processes will benefit as the data are integrated with the existing upper-air observational network and planned enhancements (e.g., autonomous aircraft, high-level jet aircraft with dropsonde capability, etc.).

In summary, spatially coherent, high-resolution coverage of upper-tropospheric winds are possible from geostationary satellite remote sensing of water vapor. WVWV are comparable in quality to operational upper-level cloud-motion vectors. However, vectors retrieved in regions lacking in definitive moisture structure are slightly less reliable, and the ability to obtain trackable information in extremely dry air masses and regions of strong subsidence is limited. The individual vectors represent single-level reports; however, upper-level vertical wind profiles are possible if multispectral observations are employed (currently possible only from GOES). The WVWV applications reported here have demonstrated the potential for analysis impact and make a strong case for enhanced remote sensing capabilities such as the Laser Atmospheric Wind Sounder (Baker et al. 1995), and the Geostationary High-Resolution Interferometer Sounder (Smith et al. 1990) that are currently under consideration. Future improvements to the GOES system will include additional WV channels in the 7$7.5-\mu \mathrm{m}$ region on the imager, with improved horizontal resolution and radiometric precision.

The next Indian satellite INSAT (to be deployed in 1997 or 1998) will join the geostationary meteorological satellite fleet containing a water vapor imager. With the exception of polar regions, global highdensity WVWV will then be possible. The international standardization and optimization of operational processing methods to enable coherent intersatellite data fusion is an objective of the World Meteorological Organization.

Acknowledgments. The second author, Dr. Christopher Hayden, recently retired from 30 years of service at NOAA. The remaining authors gratefully acknowledge Kit's accomplishments and contributions to satellite meteorology. Special thanks are extended to Jeff Hawkins of NRL-Monterey and Major Roger Edson of JTWC for their enthusiastic support, and to Brian Soden 
of GFDL and Robert Aune of CIMSS for their diligent efforts with the data assimilation studies. Robert Merrill and Tim Olander are gratefully acknowledged for contributions to the code development and data processing; Tim Schmit and Paul Vandelst for supplying figures; and John Molinari, Tom Achtor, and two anonymous referees for reviewing and improving the manuscript. Finally, the vision of Dr. Verner Suomi, recently deceased, will continue to be felt within the research dedicated to the advancement of satellite meteorology at SSEC CIMSS. This study was funded primarily by NOAA Grant 50WCNE-306075, with partial support from NASA Contract NAG8-974 and NRL Contract N00014-95-C-6017 (Spawar PE 0603207N and ONR PE $0602435 \mathrm{~N}$ ).

\section{References}

Avila, L. A., and E. N. Rappaport, 1996: Atlantic hurricane season of 1994. Mon. Wea. Rev., 124, 1558-1578.

Baker, W. E., and Coauthors, 1995: Lidar-measured winds from space: A key component for weather and climate prediction. Bull. Amer. Meteor. Soc., 76, 869-888.

DeMaria, M., 1995: Another look at the effect of vertical shear on tropical cyclone intensity change. Preprints, 21 st Conf. Hurricanes and Tropical Meteorology, Miami, FL, Amer. Meteor. Soc., 323-325.

- and J. Kaplan, 1994: A statistical hurricane intensity prediction scheme (SHIPS) for the Atlantic basin. Wea. Forecasting, 9, 209-220.

Dong, K., and C. J. Neumann, 1986: The relationship between tropical cyclone motion and environmental geostrophic flows. Mon. Wea. Rev., 114, 115-122.

Eigenwillig, N., and H. Fischer, 1982: Determination of midtropospheric wind vectors by tracking pure water vapor structure in METEOSAT water vapor image sequences. Bull. Amer. Meteor. Soc., 63, 44-57.

Franklin, J. L., S. E. Feuer, J. Kaplan, and S. D. Aberson, 1996: Tropical cyclone motion and surrounding flow relationships: Searching for beta gyres in omega dropwindsonde datasets. Mon. Wea. Rev., 124, 64-84.

Goerss, J. S., and R. A. Jeffries, 1994: Assimilation of synthetic tropical cyclone observations into the navy operational global atmospheric prediction system. Wea. Forecasting, 9, $557-576$.

Gray, W. M., 1968: Global view of the origin of tropical disturbances and storms. Mon. Wea. Rev., 96, 669-700.

Hayden, C. M., and T. R. Stewart, 1987: An update on cloud and water vapor tracers for providing wind estimates. $E x$ tended Abstracts, Sixth Symp. on Meteorological Observation and Instrumentation, New Orleans, LA, Amer. Meteor. Soc., 70-75.

, and R. J. Purser, 1988: Three-dimensional recursive filter objective analysis of meteorological fields. Preprints, Eighth Conf. on Numerical Weather Prediction, Baltimore, MD, Amer. Meteor. Soc., 185-190.

- , and C. S. Velden, 1991: Quality control and assimilation experiments with satellite derived wind estimates. Preprints, Ninth Conf. on Numerical Weather Prediction, Denver, CO, Amer. Meteor. Soc., 19-23.
, and R. J. Purser, 1995: Recursive filter objective analysis of meteorological fields: Applications to NESDIS operational processing. J. Appl. Meteor., 34, 3-15.

Hoehne, W. E., 1980: Precision of National Weather Service upper air measurements. NOAA Tech. Memo. NWS T\&ED-16, $23 \mathrm{pp}$.

Holmlund, K., 1993: Operational water vapor wind vectors from Meteosat imagery. Second Workshop on Wind Extraction from Operational Satellite Data, Tokyo, Japan, EUMETSAT, 7784.

- 1995: Half hourly wind data from satellite derived water vapour measurements. Adv. Space. Res., 16, (10)59-(10)68.

Hoskins, B. J., M. E. McIntyre, and A. W. Robertson, 1985: On the use and significance of isentropic potential vorticity maps. Quart. J. Roy. Meteor. Soc., 111, 877-946.

Jones, S. C., 1995: The evolution of vortices in vertical shear: Part 1: Initially barotropic vortices. Quart. J. Roy. Meteor. Soc., 121, 821-851.

Kurihara, Y., M. A. Bender, R. E. Tuleya, and R. J. Ross, 1995: Improvements in the GFDL hurricane prediction system. Mon. Wea. Rev., 123, 2791-2802.

Laurent, H., 1993: Wind extraction from Meteosat water vapor channel image data. J. Appl. Meteor., 32, 1124-1133.

LeMarshall, J. F., W. L. Smith, and G.M. Callan, 1985: Hurricane Debby - An illustration of the complementary nature of VAS soundings and cloud and water vapor motion winds. Bull. Amer. Meteor. Soc., 66, 258-263.

Menzel, W. P., and J. F. W. Purdom, 1994: Introducing GOESI: The first of a new generation of geostationary operational environmental satellites. Bull. Amer. Meteor. Soc, 75, 757780.

Merrill, R. T., 1989: Advances in the automated production of wind estimates from geostationary satellite imagery. Preprints, Fourth Conf. on Satellite Meteorology and Oceanography, San Diego, CA, Amer. Meteor. Soc., 246-249.

—, W. P. Menzel, W. Baker, J. Lynch, and E. Legg, 1991: A report on the recent demonstration of NOAA's upgraded capability to derive cloud motion satellite winds. Bull. Amer. Meteor. Soc., 72, 372-376.

Molinari, J., S. Skubis, and D. Vallaro, 1995: A potential vorticity view of hurricane-trough interactions. Preprints, 21 st Conf. Hurricane and Tropical Meteorology, Miami, FL, Amer. Meteor. Soc., 330-331.

Nieman, S. J., W. P. Menzel, C. M. Hayden, D. Gray, S. Wanzong, C. S. Velden, and J. Daniels, 1997: Fully automated clouddrift winds in NESDIS operations. Bull. Amer. Meteor. Soc., in press.

Pike, A. C., 1985: Geopotential heights and thicknesses as predictors of Atlantic tropical cyclone motion and intensity. Mon. Wea. Rev., 113, 931-939.

Schmetz, J., and O. M. Turpeinen, 1988: Estimation of the upper tropospheric relative humidity field from Meteosat water vapor image data. J. Appl. Meteor., 27, 889-899.

_ and L. van de Berg, 1994: Upper tropospheric humidity observations from Meteosat compared with short-term forecast fields. Geophys. Res. Lett., 21, 573-576.

- , and Coauthors, 1995: Monthly mean large-scale analyses of upper-tropospheric humidity and wind field divergence derived from three geostationary satellites. Bull. Amer. Meteor. Soc., 76, 1577-1584. 
Secretariat of Range Commanders Council, 1981: Meteorological data error estimates: 4th revision. Document 110-81, $24 \mathrm{pp}$. [Available from White Sands Missile Range. White Sands. NM 88002.]

Smith, W. I., and Coauthors, 1990: GHIS-The GOES high-resolution interferometer sounder. J. Appl. Meteor., 29, 1189 1204.

Soden, B., and F. P. Bretherton, 1993: Upper tropospheric relative humidity from GOES 6.7 micron channel: Method and climatology for July 1987. J. Geophys. Res., 98, $16669-$ 16688 .

Stewart, T. R., C. M. Hayden, and W. L. Smith, 1985: A note on water-vapor wind tracking using VAS data on McIDAS. Bull. Amer. Meteor. Soc., 66, 1111-1115.

van de Berg, L., A. Pyomjamsri, and J. Schmetz, 1991: Monthly mean upper-tropospheric humidities in cloud-free areas from METEOSAT observations. Int. J. Climatol., 11, 819-826.

Velden, C. S., 1987: Satellite observations of Hurricane Elena (1985) using the VAS $6.7 \mu \mathrm{m}$ "water-vapor" channel. Bull. Amer. Meteor. Soc., 68, 210-215.

, 1990: The impact of satellite-derived winds on hurricane analysis and track forecasting. Preprints, Fifth Conf. on Satellite Meteorology and Oceanography, London, England, Amer. Meteor. Soc., 215-219.
1993: Investigation of water vapor motion winds from geostationary satellites. Second Workshop on Wind Extraction from Operational Satellite Data, Tokyo, Japan, EUMETSAT, 99-104.

, 1996: Winds derived from geostationary satellite moisture channel observations: Applications and impact on numerical weather prediction. Meteor. Atmos. Physics, 60, 37-46.

- and L. M. Leslie, 1991: The basic relationship between tropical cyclone intensity and the depth of the environmental steering layer in the Australian region. Wea. Forecasting. 6, 244-253.

C. M. Hayden, W. P. Menzel, J. L. Franklin, and J. Lynch, 1992: The impact of satellite-derived winds on numerical hurricane track forecasting. Wea. Forecasting, 7, 107-118.

, S. J. Nieman and S. Wanzong, 1994: Investigation of water vapor motion winds from geostationary satellites. Preprints, Seventh Conf. on Satellite Meteorology and Oceanography, Monterey, CA, Amer. Meteor. Soc.. 360-363.

Weldon, R. B., and S. J. Holmes, 1991: Water vapor imagery: Interpretation and applications to weather analysis and forecasting. NOAA Tech. Rep. NESDIS 67, 213 pp. |Available from NOAA Science Center, 5200 Auth Rd., Camp Springs, MD 20748.] 
